\title{
STABILIZATION OF SECOND ORDER EVOLUTION EQUATIONS WITH UNBOUNDED FEEDBACK WITH DELAY
}

\author{
Serge Nicaise $^{1}$ And Julie VAlein ${ }^{1}$
}

\begin{abstract}
We consider abstract second order evolution equations with unbounded feedback with delay. Existence results are obtained under some realistic assumptions. Sufficient and explicit conditions are derived that guarantee the exponential or polynomial stability. Some new examples that enter into our abstract framework are presented.
\end{abstract}

Mathematics Subject Classification. 93D15, 93C20.

Received April 16, 2008. Revised September 19, 2008 and December 1st, 2008.

Published online April 21, 2009.

\section{INTRODUCTION}

Time-delay often appears in many biological, electrical engineering systems and mechanical applications $[1,11,21]$, and in many cases, in particular for distributed parameter systems, even arbitrarily small delays in the feedback may destabilize the system, see e.g. $[8-10,12,15-17,20,23]$. The stability issue of systems with delay is, therefore, of theoretical and practical importance.

We further remark that some techniques developed recently $[16,17]$ in order to obtain some existence results and decay rates have some similarities. We therefore propose to consider an abstract setting as large as possible in order to contain a quite large class of problems with time delay feedbacks. In a second step we prove existence and stability results in this setting under realistic assumptions. Finally in order to show the usefulness of our approach, we give some examples where our abstract framework can be applied. For a similar approach, we refer to the paper in preparation [4]. Without delay such an approach was developed in [3].

Before going on, let us present our abstract framework. Let $H$ be a real Hilbert space with norm and inner product denoted respectively by $\|\cdot\|_{H}$ and $(., .)_{H}$. Let $A: D(A) \rightarrow H$ be a self-adjoint positive operator with a compact inverse in $H$. Let $V:=D\left(A^{\frac{1}{2}}\right)$ be the domain of $A^{\frac{1}{2}}$. Denote by $D\left(A^{\frac{1}{2}}\right)^{\prime}$ the dual space of $D\left(A^{\frac{1}{2}}\right)$ obtained by means of the inner product in $H$.

Further, for $i=1,2$, let $U_{i}$ be a real Hilbert space (which will be identified to its dual space) with norm and inner product denoted respectively by $\|\cdot\|_{U_{i}}$ and $(.,)_{U_{i}}$ and let $B_{i} \in \mathcal{L}\left(U_{i}, D\left(A^{\frac{1}{2}}\right)^{\prime}\right)$.

\footnotetext{
Keywords and phrases. Second order evolution equations, wave equations, delay, stabilization functional.

1 Université de Valenciennes et du Hainaut Cambrésis, LAMAV, FR CNRS 2956, Institut des Sciences et Techniques of Valenciennes, 59313 Valenciennes Cedex 9, France. Serge.Nicaise@univ-valenciennes.fr;

Julie.Valein@univ-valenciennes.fr
} 
We consider the system described by

$$
\left\{\begin{array}{c}
\ddot{\omega}(t)+A \omega(t)+B_{1} u_{1}(t)+B_{2} u_{2}(t-\tau)=0, t>0 \\
\omega(0)=\omega_{0}, \dot{\omega}(0)=\omega_{1} \\
u_{2}(t-\tau)=f^{0}(t-\tau), 0<t<\tau
\end{array}\right.
$$

where $t \in[0, \infty)$ represents the time, $\tau$ is a positive constant which represents the delay, $\omega:[0, \infty) \rightarrow H$ is the state of the system and $u_{1} \in L^{2}\left([0, \infty), U_{1}\right), u_{2} \in L^{2}\left([-\tau, \infty), U_{2}\right)$ are the input functions. Most of the linear equations modelling the vibrations of elastic structures with distributed control with delay can be written in the form (1.1), where $\omega$ stands for the displacement field.

In many problems, coming in particular from elasticity, the input $u_{i}$ are given in the feedback form $u_{i}(t)=$ $B_{i}^{*} \dot{\omega}(t)$, which corresponds to colocated actuators and sensors. We obtain in this way the closed loop system

$$
\left\{\begin{array}{c}
\ddot{\omega}(t)+A \omega(t)+B_{1} B_{1}^{*} \dot{\omega}(t)+B_{2} B_{2}^{*} \dot{\omega}(t-\tau)=0, t>0 \\
\omega(0)=\omega_{0}, \dot{\omega}(0)=\omega_{1} \\
B_{2}^{*} \dot{\omega}(t-\tau)=f^{0}(t-\tau), 0<t<\tau
\end{array}\right.
$$

The first natural question is the well-posedness of this system. In Section 2 we will give a sufficient condition that guarantees that this system (1.2) is well-posed, where we closely follow the approach developed in [16] for the wave equation. Secondly, we may ask if this system is dissipative. We show in Section 3 that the condition

$$
\exists 0<\alpha<1, \forall u \in V,\left\|B_{2}^{*} u\right\|_{U_{2}}^{2} \leq \alpha\left\|B_{1}^{*} u\right\|_{U_{1}}^{2}
$$

guarantees the energy is decreasing; under this condition, using a result from [5] (see also [22]) we pertain a necessary and sufficient condition for the decay to zero of the energy. Note that this last condition is independent of the delay and therefore under the condition (1.3), our system is strongly stable if and only if the same system without delay is strongly stable. Note further that if (1.3) is not satisfied, there exist cases where some instabilities may appear (see $[16,17,23]$ for the wave equation). Hence this assumption seems realistic.

In a third step, again under the condition (1.3) and a certain boundedness assumption from [3] between the resolvent operator of $A$ and of the operators $B_{1}$ and $B_{2}$, see condition (4.1), we prove that the exponential decay of the system (1.2) follows from a certain observability estimate. Again this observability estimate is independent of the delay term $B_{2} B_{2}^{*} \dot{\omega}(t-\tau)$ and therefore, under the conditions (1.3) and (4.1), the exponential decay of the system (1.2) follows from the exponential decay of the same system without delay. Nevertheless we give the dependence of the decay rate with respect to the delay, in particular we show that if the delay increases the decay rate decreases. This is the content of Section 4. A similar analysis for the polynomial decay is performed in Section 5 by weakening the observability estimate. Again we show that if the delay increases the decay rate decreases. In view of some applications, Section 6 is devoted to the proof of these two observability estimates by using a frequency domain method and a reduction to some conditions between the eigenvectors of $A$ and the feedback operator $B_{1}^{*}$.

Finally we finish this paper by considering in Section 7 different examples where our abstract framework can be applied. To our knowledge, all the examples, with the exception of the first one, are new.

\section{WELL-POSEDNESS OF THE SYSTEM}

We aim to show that system (1.2) is well-posed. For that purpose, we use semi-group theory and an idea from [16] (see also [17]). Let us introduce the auxiliary variable $z(\rho, t)=B_{2}^{*} \dot{\omega}(t-\tau \rho)$ for $\rho \in(0,1)$ and $t>0$. 
Note that $z$ verifies the transport equation for $0<\rho<1$ and $t>0$

$$
\left\{\begin{array}{c}
\tau \frac{\partial z}{\partial t}+\frac{\partial z}{\partial \rho}=0 \\
z(0, t)=B_{2}^{*} \dot{\omega}(t) \\
z(\rho, 0)=B_{2}^{*} \dot{\omega}(-\tau \rho)=f^{0}(-\tau \rho)
\end{array}\right.
$$

Therefore, the system (1.2) is equivalent to

$$
\left\{\begin{array}{c}
\ddot{\omega}(t)+A \omega(t)+B_{1} B_{1}^{*} \dot{\omega}(t)+B_{2} z(1, t)=0, t>0 \\
\tau \frac{\partial z}{\partial t}+\frac{\partial z}{\partial \rho}=0, t>0,0<\rho<1 \\
\omega(0)=\omega_{0}, \dot{\omega}(0)=\omega_{1}, z(\rho, 0)=f^{0}(-\tau \rho), 0<\rho<1 \\
z(0, t)=B_{2}^{*} \dot{\omega}(t), t>0 .
\end{array}\right.
$$

If we introduce

$$
U:=(\omega, \dot{\omega}, z)^{T}
$$

then $U$ satisfies

$$
U^{\prime}=(\dot{\omega}, \ddot{\omega}, \dot{z})^{T}=\left(\dot{\omega},-A \omega(t)-B_{1} B_{1}^{*} \dot{\omega}(t)-B_{2} z(1, t),-\frac{1}{\tau} \frac{\partial z}{\partial \rho}\right)^{T} .
$$

Consequently the system (1.2) may be rewritten as the first order evolution equation

$$
\left\{\begin{array}{c}
U^{\prime}=\mathcal{A} U \\
U(0)=\left(\omega_{0}, \omega_{1}, f^{0}(-\tau .)\right)
\end{array}\right.
$$

where the operator $\mathcal{A}$ is defined by

$$
\mathcal{A}\left(\begin{array}{l}
\omega \\
u \\
z
\end{array}\right)=\left(\begin{array}{c}
u \\
-A \omega-B_{1} B_{1}^{*} u-B_{2} z(1) \\
-\frac{1}{\tau} \frac{\partial z}{\partial \rho}
\end{array}\right),
$$

with domain

$$
D(\mathcal{A}):=\left\{(\omega, u, z) \in V \times V \times H^{1}\left((0,1), U_{2}\right) ; z(0)=B_{2}^{*} u, A \omega+B_{1} B_{1}^{*} u+B_{2} z(1) \in H\right\} .
$$

Now, we introduce the Hilbert space

$$
\mathcal{H}=V \times H \times L^{2}\left((0,1), U_{2}\right)
$$

equipped with the usual inner product

$$
\left\langle\left(\begin{array}{c}
\omega \\
u \\
z
\end{array}\right),\left(\begin{array}{c}
\tilde{\omega} \\
\tilde{u} \\
\tilde{z}
\end{array}\right)\right\rangle=\left(A^{\frac{1}{2}} \omega, A^{\frac{1}{2}} \tilde{\omega}\right)_{H}+(u, \tilde{u})_{H}+\int_{0}^{1}(z(\rho), \tilde{z}(\rho))_{U_{2}} \mathrm{~d} \rho .
$$

Let us suppose now that

$$
\exists 0<\alpha \leq 1, \forall u \in V,\left\|B_{2}^{*} u\right\|_{U_{2}}^{2} \leq \alpha\left\|B_{1}^{*} u\right\|_{U_{1}}^{2} .
$$

Under this condition, we will show that the operator $\mathcal{A}$ generates a $C_{0}$-semigroup in $\mathcal{H}$. 
For that purpose, we choose a positive real number $\xi$ such that

$$
1 \leq \xi \leq \frac{2}{\alpha}-1 .
$$

This constant exists because $0<\alpha \leq 1$.

We now introduce the following inner product on $\mathcal{H}$

$$
\left\langle\left(\begin{array}{c}
\omega \\
u \\
z
\end{array}\right),\left(\begin{array}{c}
\tilde{\omega} \\
\tilde{u} \\
\tilde{z}
\end{array}\right)\right\rangle_{\mathcal{H}}=\left(A^{\frac{1}{2}} \omega, A^{\frac{1}{2}} \tilde{\omega}\right)_{H}+(u, \tilde{u})_{H}+\tau \xi \int_{0}^{1}(z(\rho), \tilde{z}(\rho))_{U_{2}} \mathrm{~d} \rho .
$$

This new inner product is clearly equivalent to the usual inner product (2.4) on $\mathcal{H}$.

Theorem 2.1. Under the assumption (2.5), for an initial datum $U_{0} \in \mathcal{H}$, there exists a unique solution $U \in C([0,+\infty), \mathcal{H})$ to system $(2.3)$. Moreover, if $U_{0} \in D(\mathcal{A})$, then

$$
U \in C([0,+\infty), D(\mathcal{A})) \cap C^{1}([0,+\infty), \mathcal{H}) .
$$

Proof. By Lumer-Phillips' theorem, it suffices to show that $\mathcal{A}$ is m-dissipative (see Def. 3.3.1 and Thms. 1.4.3 and 1.4.6 of [18]).

We first prove that $\mathcal{A}$ is dissipative. Take $U=(\omega, u, z)^{\top} \in D(\mathcal{A})$. Then

$$
\begin{aligned}
\langle\mathcal{A} U, U\rangle_{\mathcal{H}} & =\left\langle\left(\begin{array}{c}
u \\
-A \omega-B_{1} B_{1}^{*} u-B_{2} z(1) \\
-\frac{1}{\tau} \frac{\partial z}{\partial \rho}
\end{array}\right),\left(\begin{array}{c}
\omega \\
u \\
z
\end{array}\right)\right\rangle_{\mathcal{H}} \\
& =\left(A^{\frac{1}{2}} u, A^{\frac{1}{2}} \omega\right)_{H}-\left(A \omega+B_{1} B_{1}^{*} u+B_{2} z(1), u\right)_{H}-\xi \int_{0}^{1}\left(\frac{\partial z}{\partial \rho}(\rho), z(\rho)\right)_{U_{2}} \mathrm{~d} \rho .
\end{aligned}
$$

Since $A \omega+B_{1} B_{1}^{*} u+B_{2} z(1) \in H$, we obtain

$$
\begin{aligned}
\langle\mathcal{A} U, U\rangle_{\mathcal{H}}= & \left(A^{\frac{1}{2}} u, A^{\frac{1}{2}} \omega\right)_{H}-\langle A \omega, u\rangle_{V^{\prime}, V}-\left\langle B_{1} B_{1}^{*} u, u\right\rangle_{V^{\prime}, V}-\left\langle B_{2} z(1), u\right\rangle_{V^{\prime}, V} \\
& -\xi \int_{0}^{1}\left(\frac{\partial z}{\partial \rho}(\rho), z(\rho)\right)_{U_{2}} \mathrm{~d} \rho \\
= & \langle A \omega, u\rangle_{V^{\prime}, V}-\langle A \omega, u\rangle_{V^{\prime}, V}-\left\|B_{1}^{*} u\right\|_{U_{1}}^{2}-\left(z(1), B_{2}^{*} u\right)_{U_{2}} \\
& -\xi \int_{0}^{1}\left(\frac{\partial z}{\partial \rho}(\rho), z(\rho)\right)_{U_{2}} \mathrm{~d} \rho
\end{aligned}
$$

by duality. By integrating by parts, we obtain

$$
\int_{0}^{1}\left(\frac{\partial z}{\partial \rho}(\rho), z(\rho)\right)_{U_{2}} \mathrm{~d} \rho=-\int_{0}^{1}\left(z(\rho), \frac{\partial z}{\partial \rho}(\rho)\right)_{U_{2}} \mathrm{~d} \rho+\left(\|z(1)\|_{U_{2}}^{2}-\|z(0)\|_{U_{2}}^{2}\right),
$$

and thus

$$
\int_{0}^{1}\left(\frac{\partial z}{\partial \rho}(\rho), z(\rho)\right)_{U_{2}} \mathrm{~d} \rho=\frac{1}{2}\left(\|z(1)\|_{U_{2}}^{2}-\left\|B_{2}^{*} u\right\|_{U_{2}}^{2}\right) .
$$

Therefore, by Cauchy-Schwarz's inequality, we find

$$
\begin{aligned}
\langle\mathcal{A} U, U\rangle_{\mathcal{H}} & =-\left\|B_{1}^{*} u\right\|_{U_{1}}^{2}-\left(z(1), B_{2}^{*} u\right)_{U_{2}}-\frac{\xi}{2}\|z(1)\|_{U_{2}}^{2}+\frac{\xi}{2}\left\|B_{2}^{*} u\right\|_{U_{2}}^{2} \\
& \leq-\left\|B_{1}^{*} u\right\|_{U_{1}}^{2}+\left(\frac{1}{2}-\frac{\xi}{2}\right)\|z(1)\|_{U_{2}}^{2}+\left(\frac{1}{2}+\frac{\xi}{2}\right)\left\|B_{2}^{*} u\right\|_{U_{2}}^{2} .
\end{aligned}
$$


By (2.5), we obtain

$$
\langle\mathcal{A} U, U\rangle_{\mathcal{H}} \leq\left(\frac{\alpha}{2}+\frac{\xi \alpha}{2}-1\right)\left\|B_{1}^{*} u\right\|_{U_{1}}^{2}+\left(\frac{1}{2}-\frac{\xi}{2}\right)\|z(1)\|_{U_{2}}^{2}
$$

with $\frac{\alpha}{2}+\frac{\xi \alpha}{2}-1 \leq 0$ and $\frac{1}{2}-\frac{\xi}{2} \leq 0$ because $\xi$ satisfies condition (2.6). This shows that $\langle\mathcal{A} U, U\rangle_{\mathcal{H}} \leq 0$ and then the dissipativeness of $\mathcal{A}$.

Let us now prove that $\lambda I-\mathcal{A}$ is surjective for some $\lambda>0$.

Let $(f, g, h)^{T} \in \mathcal{H}$. We look for $U=(\omega, u, z)^{T} \in D(\mathcal{A})$ solution of

$$
(\lambda I-\mathcal{A})\left(\begin{array}{c}
\omega \\
u \\
z
\end{array}\right)=\left(\begin{array}{c}
f \\
g \\
h
\end{array}\right)
$$

or equivalently

$$
\left\{\begin{array}{l}
\lambda \omega-u=f \\
\lambda u+A \omega+B_{1} B_{1}^{*} u+B_{2} z(1)=g \\
\lambda z+\frac{1}{\tau} \frac{\partial z}{\partial \rho}=h .
\end{array}\right.
$$

Suppose that we have found $\omega$ with the appropriate regularity. Then, we have

$$
u=-f+\lambda \omega \in V .
$$

We can then determine $z$, indeed $z$ satisfies the differential equation

$$
\lambda z+\frac{1}{\tau} \frac{\partial z}{\partial \rho}=h
$$

and the boundary condition $z(0)=B_{2}^{*} u=-B_{2}^{*} f+\lambda B_{2}^{*} \omega$. Therefore $z$ is explicitly given by

$$
z(\rho)=\lambda B_{2}^{*} \omega \mathrm{e}^{-\lambda \tau \rho}-B_{2}^{*} f \mathrm{e}^{-\lambda \tau \rho}+\tau \mathrm{e}^{-\lambda \tau \rho} \int_{0}^{\rho} \mathrm{e}^{\lambda \tau \sigma} h(\sigma) \mathrm{d} \sigma .
$$

This means that once $\omega$ is found with the appropriate properties, we can find $z$ and $u$. In particular, we have

$$
z(1)=\lambda B_{2}^{*} \omega \mathrm{e}^{-\lambda \tau}-B_{2}^{*} f \mathrm{e}^{-\lambda \tau}+\tau \mathrm{e}^{-\lambda \tau} \int_{0}^{1} \mathrm{e}^{\lambda \tau \sigma} h(\sigma) \mathrm{d} \sigma=\lambda B_{2}^{*} \omega \mathrm{e}^{-\lambda \tau}+z^{0},
$$

where $z^{0}=-B_{2}^{*} f \mathrm{e}^{-\lambda \tau}+\tau \mathrm{e}^{-\lambda \tau} \int_{0}^{1} \mathrm{e}^{\lambda \tau \sigma} h(\sigma) \mathrm{d} \sigma$ is a fixed element of $U_{2}$ depending only on $f$ and $h$.

It remains to find $\omega$. By (2.7), $\omega$ must satisfy

$$
\lambda^{2} \omega+A \omega+\lambda B_{1} B_{1}^{*} \omega+B_{2} z(1)=g+B_{1} B_{1}^{*} f+\lambda f,
$$

and thus by (2.8),

$$
\lambda^{2} \omega+A \omega+\lambda B_{1} B_{1}^{*} \omega+\lambda \mathrm{e}^{-\lambda \tau} B_{2} B_{2}^{*} \omega=g+B_{1} B_{1}^{*} f+\lambda f-B_{2} z^{0}=: q,
$$

where $q \in V^{\prime}$. We take then the duality brackets $\langle., .\rangle_{V^{\prime}, V}$ with $\phi \in V$ :

$$
\left\langle\lambda^{2} \omega+A \omega+\lambda B_{1} B_{1}^{*} \omega+\lambda \mathrm{e}^{-\lambda \tau} B_{2} B_{2}^{*} \omega, \phi\right\rangle_{V^{\prime}, V}=\langle q, \phi\rangle_{V^{\prime}, V}
$$


Moreover:

$$
\begin{aligned}
& \left\langle\lambda^{2} \omega+A \omega+\lambda B_{1} B_{1}^{*} \omega+\lambda \mathrm{e}^{-\lambda \tau} B_{2} B_{2}^{*} \omega, \phi\right\rangle_{V^{\prime}, V} \\
& \quad=\lambda^{2}\langle\omega, \phi\rangle_{V^{\prime}, V}+\langle A \omega, \phi\rangle_{V^{\prime}, V}+\lambda\left(\left\langle B_{1} B_{1}^{*} \omega, \phi\right\rangle_{V^{\prime}, V}+\mathrm{e}^{-\lambda \tau}\left\langle B_{2} B_{2}^{*} \omega, \phi\right\rangle_{V^{\prime}, V}\right) \\
& =\lambda^{2}(\omega, \phi)_{H}+\left(A^{\frac{1}{2}} \omega, A^{\frac{1}{2}} \phi\right)_{H}+\lambda\left(\left(B_{1}^{*} \omega, B_{1}^{*} \phi\right)_{U_{1}}+\mathrm{e}^{-\lambda \tau}\left(B_{2}^{*} \omega, B_{2}^{*} \phi\right)_{U_{2}}\right)
\end{aligned}
$$

because $\omega \in V \subset H$. Consequently, we arrive at the problem

$$
\lambda^{2}(\omega, \phi)_{H}+\left(A^{\frac{1}{2}} \omega, A^{\frac{1}{2}} \phi\right)_{H}+\lambda\left(\left(B_{1}^{*} \omega, B_{1}^{*} \phi\right)_{U_{1}}+\mathrm{e}^{-\lambda \tau}\left(B_{2}^{*} \omega, B_{2}^{*} \phi\right)_{U_{2}}\right)=\langle q, \phi\rangle_{V^{\prime}, V} .
$$

The left hand side of (2.9) is continuous and coercive on $V$. Indeed, we have

$$
\begin{aligned}
& \left|\lambda^{2}(\omega, \phi)_{H}+\left(A^{\frac{1}{2}} \omega, A^{\frac{1}{2}} \phi\right)_{H}+\lambda\left(\left(B_{1}^{*} \omega, B_{1}^{*} \phi\right)_{U_{1}}+\mathrm{e}^{-\lambda \tau}\left(B_{2}^{*} \omega, B_{2}^{*} \phi\right)_{U_{2}}\right)\right| \\
& \quad \leq \lambda^{2}\|\omega\|_{H}\|\phi\|_{H}+\left\|A^{\frac{1}{2}} \omega\right\|_{H}\left\|A^{\frac{1}{2}} \phi\right\|_{H}+\lambda\left(\left\|B_{1}^{*} \omega\right\|_{U_{1}}\left\|B_{1}^{*} \phi\right\|_{U_{1}}+\mathrm{e}^{-\lambda \tau}\left\|B_{2}^{*} \omega\right\|_{U_{2}}\left\|B_{2}^{*} \phi\right\|_{U_{2}}\right) \\
& \quad \leq C \lambda^{2}\|\omega\|_{V}\|\phi\|_{H}+\left\|A^{\frac{1}{2}}\right\|^{2}\|\omega\|_{V}\|\phi\|_{V}+\lambda\left(\left\|B_{1}^{*}\right\|_{\mathcal{L}\left(V, U_{1}\right)}^{2}\|\omega\|_{V}\|\phi\|_{V}+\mathrm{e}^{-\lambda \tau}\left\|B_{2}^{*}\right\|_{\mathcal{L}\left(V, U_{2}\right)}^{2}\|\omega\|_{V}\|\phi\|_{V}\right) \\
& \quad \leq C\|\omega\|_{V}\|\phi\|_{V},
\end{aligned}
$$

and for $\phi=\omega \in V$

$$
\lambda^{2}\|\omega\|_{H}^{2}+\left(A^{\frac{1}{2}} \omega, A^{\frac{1}{2}} \omega\right)_{H}+\lambda\left(\left\|B_{1}^{*} \omega\right\|_{U_{1}}^{2}+\mathrm{e}^{-\lambda \tau}\left\|B_{2}^{*} \omega\right\|_{U_{2}}^{2}\right) \geq\left\|A^{\frac{1}{2}} \omega\right\|_{H}^{2} \geq C\|\omega\|_{V}^{2}
$$

Therefore, this problem (2.9) has a unique solution $\omega \in V$ by Lax-Milgram's lemma. Moreover $A \omega+B_{1} B_{1}^{*} u+$ $B_{2} z(1)=g+\lambda f-\lambda^{2} \omega \in H$. In summary, we have found $(\omega, u, z)^{T} \in D(\mathcal{A})$ satisfying $(2.7)$.

Remark 2.2. We deduce from Theorem 2.1 that $D(\mathcal{A})$ is dense in $\mathcal{H}$ (see [18]).

Remark 2.3. For initial data $\left(\omega_{0}, \omega_{1}, f^{0}(-\tau .)\right)^{T}$ in $D(\mathcal{A})$, we easily show that the solution $(\omega(t), u(t), z(t))^{T}=$ $T(t)\left(\omega_{0}, \omega_{1}, f^{0}(-\tau .)\right)^{T}$, where $T(t)$ is the semigroup generated by $\mathcal{A}$, is indeed solution of (1.2) in the sense that

$$
u(t)=\dot{\omega}(t),
$$

and

and $\omega$ satisfies (1.2).

$$
z(\rho, t)=B_{2}^{*}(t-\tau \rho)
$$

\section{The EnERGy}

We now restrict hypothesis (2.5) to obtain the decay of the energy. Namely, we suppose that (1.3) holds, namely

$$
\exists 0<\alpha<1, \forall u \in V,\left\|B_{2}^{*} u\right\|_{U_{2}}^{2} \leq \alpha\left\|B_{1}^{*} u\right\|_{U_{1}}^{2}
$$

For an initial datum $\left(\omega_{0}, \omega_{1}, f^{0}(-\tau .)\right)^{T} \in \mathcal{H}$, Theorem 2.1 guarantees the existence of a weak solution $(\omega(t), u(t), z(t))^{T}=T(t)\left(\omega_{0}, \omega_{1}, f^{0}(-\tau .)\right)^{T}$. Hence the associated energy (which corresponds to the inner product on $\mathcal{H})$ is defined by

$$
E(t):=\frac{1}{2}\left(\left\|A^{\frac{1}{2}} \omega(t)\right\|_{H}^{2}+\|u(t)\|_{H}^{2}+\tau \xi \int_{0}^{1}\|z(\rho, t)\|_{U_{2}}^{2} \mathrm{~d} \rho\right)
$$


where $\xi$ is a positive constant satisfying

$$
1<\xi<\frac{2}{\alpha}-1
$$

that exists because $0<\alpha<1$.

Note that by Remark 2.3 for initial data in $D(\mathcal{A})$, this energy takes the form

$$
E(t):=\frac{1}{2}\left(\left\|A^{\frac{1}{2}} \omega\right\|_{H}^{2}+\|\dot{\omega}\|_{H}^{2}+\tau \xi \int_{0}^{1}\left\|B_{2}^{*} \dot{\omega}(t-\tau \rho)\right\|_{U_{2}}^{2} \mathrm{~d} \rho\right)
$$

\subsection{Decay of the energy}

Proposition 3.1. If (1.3) holds, then for all $\left(\omega_{0}, \omega_{1}, f^{0}(-\tau .)\right)^{T} \in D(\mathcal{A})$, the energy of the corresponding regular solution of $(1.2)$ (i.e. $\left.\left(\omega, \dot{\omega}, B_{2} \dot{\omega}(t-\tau \rho)\right)^{T} \in C([0,+\infty), D(\mathcal{A})) \cap C^{1}([0,+\infty), \mathcal{H})\right)$ is non-increasing and there exist two positive constants $C_{1}$ and $C_{2}$ depending only on $\alpha$ and $\xi$ such that

$$
-C_{2}\left(\left\|B_{1}^{*} \dot{\omega}(t)\right\|_{U_{1}}^{2}+\left\|B_{2}^{*} \dot{\omega}(t-\tau)\right\|_{U_{2}}^{2}\right) \leq E^{\prime}(t) \leq-C_{1}\left(\left\|B_{1}^{*} \dot{\omega}(t)\right\|_{U_{1}}^{2}+\left\|B_{2}^{*} \dot{\omega}(t-\tau)\right\|_{U_{2}}^{2}\right) .
$$

Proof. Deriving (3.2), we obtain

$$
\begin{aligned}
E^{\prime}(t)= & \left(A^{\frac{1}{2}} \omega, A^{\frac{1}{2}} \dot{\omega}\right)_{H}+(\dot{\omega}, \ddot{\omega})_{H}+\tau \xi \int_{0}^{1}\left(B_{2}^{*} \dot{\omega}(t-\tau \rho), B_{2}^{*} \ddot{\omega}(t-\tau \rho)\right)_{U_{2}} \mathrm{~d} \rho \\
= & \langle A \omega, \dot{\omega}\rangle_{V^{\prime}, V}-\left(\dot{\omega}, A \omega+B_{1} B_{1}^{*} \dot{\omega}+B_{2} B_{2}^{*} \dot{\omega}(t-\tau)\right)_{H} \\
& +\xi \tau \int_{0}^{1}\left(B_{2}^{*} \dot{\omega}(t-\tau \rho), B_{2}^{*} \ddot{\omega}(t-\tau \rho)\right)_{U_{2}} \mathrm{~d} \rho \\
= & \langle A \omega, \dot{\omega}\rangle_{V^{\prime}, V}-\left\langle\dot{\omega}, A \omega+B_{1} B_{1}^{*} \dot{\omega}+B_{2} B_{2}^{*} \dot{\omega}(t-\tau)\right\rangle_{V, V^{\prime}} \\
& +\xi \tau \int_{0}^{1}\left(B_{2}^{*} \dot{\omega}(t-\tau \rho), B_{2}^{*} \ddot{\omega}(t-\tau \rho)\right)_{U_{2}} \mathrm{~d} \rho,
\end{aligned}
$$

because $A \omega+B_{1} B_{1}^{*} \dot{\omega}+B_{2} B_{2}^{*} \dot{\omega}(t-\tau) \in H$. Then

$$
\begin{aligned}
E^{\prime}(t)= & \langle A \omega, \dot{\omega}\rangle_{V^{\prime}, V}-\langle\dot{\omega}, A \omega\rangle_{V, V^{\prime}}-\left\langle\dot{\omega}, B_{1} B_{1}^{*} \dot{\omega}\right\rangle_{V, V^{\prime}}-\left\langle\dot{\omega}, B_{2} B_{2}^{*} \dot{\omega}(t-\tau)\right\rangle_{V, V^{\prime}} \\
& +\xi \tau \int_{0}^{1}\left(B_{2}^{*} \dot{\omega}(t-\tau \rho), B_{2}^{*} \ddot{\omega}(t-\tau \rho)\right)_{U_{2}} \mathrm{~d} \rho \\
= & -\left\|B_{1}^{*} \dot{\omega}\right\|_{U_{1}}^{2}-\left(B_{2}^{*} \dot{\omega}, B_{2}^{*} \dot{\omega}(t-\tau)\right)_{U_{2}} \\
& +\xi \tau \int_{0}^{1}\left(B_{2}^{*} \dot{\omega}(t-\tau \rho), B_{2}^{*} \ddot{\omega}(t-\tau \rho)\right)_{U_{2}} \mathrm{~d} \rho
\end{aligned}
$$

Moreover, recalling that $z(\rho, t)=B_{2}^{*} \dot{\omega}(t-\tau \rho)$, we see that

$$
\begin{aligned}
\int_{0}^{1}\left(B_{2}^{*} \dot{\omega}(t-\tau \rho), B_{2}^{*} \ddot{\omega}(t-\tau \rho)\right)_{U_{2}} \mathrm{~d} \rho & =\int_{0}^{1}\left(z(\rho, t), \frac{\partial z}{\partial t}(\rho, t)\right)_{U_{2}} \mathrm{~d} \rho \\
& =-\frac{1}{\tau} \int_{0}^{1}\left(z(\rho, t), \frac{\partial z}{\partial \rho}(\rho, t)\right)_{U_{2}} \mathrm{~d} \rho
\end{aligned}
$$


because $\frac{\partial z}{\partial \rho}(\rho, t)=-\tau \frac{\partial z}{\partial t}(\rho, t)$. Then, we have

$$
\begin{aligned}
\int_{0}^{1}\left(B_{2}^{*} \dot{\omega}(t-\tau \rho), B_{2}^{*} \ddot{\omega}(t-\tau \rho)\right)_{U_{2}} \mathrm{~d} \rho & =-\frac{1}{2 \tau} \int_{0}^{1} \frac{\partial}{\partial \rho}\|z(\rho, t)\|_{U_{2}}^{2} \mathrm{~d} \rho \\
& =-\frac{1}{2 \tau}\left(\|z(1, t)\|_{U_{2}}^{2}-\|z(0, t)\|_{U_{2}}^{2}\right) \\
& =-\frac{1}{2 \tau}\left(\left\|B_{2}^{*} \dot{\omega}(t-\tau)\right\|_{U_{2}}^{2}-\left\|B_{2}^{*} \dot{\omega}(t)\right\|_{U_{2}}^{2}\right) .
\end{aligned}
$$

Consequently,

$$
E^{\prime}(t)=-\left\|B_{1}^{*} \dot{\omega}\right\|_{U_{1}}^{2}-\left(B_{2}^{*} \dot{\omega}, B_{2}^{*} \dot{\omega}(t-\tau)\right)_{U_{2}}-\frac{\xi}{2}\left\|B_{2}^{*} \dot{\omega}(t-\tau)\right\|_{U_{2}}^{2}+\frac{\xi}{2}\left\|B_{2}^{*} \dot{\omega}(t)\right\|_{U_{2}}^{2} .
$$

Cauchy-Schwarz's inequality yields

$$
E^{\prime}(t) \leq-\left\|B_{1}^{*} \dot{\omega}\right\|_{U_{1}}^{2}+\left(\frac{1}{2}+\frac{\xi}{2}\right)\left\|B_{2}^{*} \dot{\omega}(t)\right\|_{U_{2}}^{2}+\left(\frac{1}{2}-\frac{\xi}{2}\right)\left\|B_{2}^{*} \dot{\omega}(t-\tau)\right\|_{U_{2}}^{2}
$$

and

$$
E^{\prime}(t) \geq-\left\|B_{1}^{*} \dot{\omega}\right\|_{U_{1}}^{2}+\left(-\frac{1}{2}+\frac{\xi}{2}\right)\left\|B_{2}^{*} \dot{\omega}(t)\right\|_{U_{2}}^{2}-\left(\frac{1}{2}+\frac{\xi}{2}\right)\left\|B_{2}^{*} \dot{\omega}(t-\tau)\right\|_{U_{2}}^{2} .
$$

Therefore, by (1.3), these estimates leads to

$$
E^{\prime}(t) \leq-C_{1}\left(\left\|B_{1}^{*} \dot{\omega}(t)\right\|_{U_{1}}^{2}+\left\|B_{2}^{*} \dot{\omega}(t-\tau)\right\|_{U_{2}}^{2}\right)
$$

with

$$
C_{1}=\min \left\{\left(1-\frac{\xi \alpha}{2}-\frac{\alpha}{2}\right),\left(\frac{\xi}{2}-\frac{1}{2}\right)\right\}
$$

which is positive according to the assumption (3.1), and to

$$
E^{\prime}(t) \geq-C_{2}\left(\left\|B_{1}^{*} \dot{\omega}\right\|_{U_{1}}^{2}+\left\|B_{2}^{*} \dot{\omega}(t-\tau)\right\|_{U_{2}}^{2}\right)
$$

with

$$
C_{2}=\max \left\{1, \frac{\xi+1}{2}\right\}
$$

which is also positive.

Remark 3.2. Integrating the expression (3.3) between 0 and $T$, we obtain

$$
\int_{0}^{T}\left(\left\|B_{1}^{*} \dot{\omega}(t)\right\|_{U_{1}}^{2}+\left\|B_{2}^{*} \dot{\omega}(t-\tau)\right\|_{U_{2}}^{2}\right) \mathrm{d} t \leq \frac{1}{C_{1}}(E(0)-E(T)) \leq \frac{1}{C_{1}} E(0) .
$$

This estimate implies that $B_{1}^{*} \dot{\omega}(.) \in L^{2}\left((0, T), U_{1}\right)$ and $B_{2}^{*} \dot{\omega}(.-\tau) \in L^{2}\left((0, T), U_{2}\right)$.

Remark 3.3. If (1.3) is not satisfied, there exist cases where instabilities may appear, see $[16,17,23]$ for the wave equation. Hence this condition appears to be quite realistic. 


\subsection{Decay of the energy to 0}

We give a necessary and sufficient condition that guarantees the decay to 0 of the energy.

Proposition 3.4. Assume that (1.3) holds. Then, for all initial data in $\mathcal{H}$, we have

$$
\lim _{t \rightarrow \infty} E(t)=0
$$

if and only if for any (non zero) eigenvector $\varphi \in D(A)$ of $A$, we have

$$
B_{1}^{*} \varphi \neq 0 .
$$

Remark 3.5. Notice that this necessary and sufficient condition is the same than in the case without delay (see [22]) and therefore, the system (1.2) with delay is strongly stable (i.e. the energy tends to zero) if and only if the system without delay (i.e. for $B_{2}=0$ ) is strongly stable.

Proof. $\Leftrightarrow$ Let us show that (3.5) implies (3.4). For that purpose we closely follow [22].

First, we show that $\mathcal{A}$ has no eigenvalue on the imaginary axis. If it is not the case, let $\mathrm{i} \omega$ be an eigenvalue of $\mathcal{A}$ where $\omega \in \mathbb{R}$. Let $\varphi$ be an eigenvector associated with $\mathrm{i} \omega$. Then $\varphi$ is of the form

$$
\varphi=\left(\begin{array}{c}
z \\
\mathrm{i} \omega z \\
\mathrm{i} \omega \mathrm{e}^{-\mathrm{i} \omega \tau \rho} B_{2}^{*} z
\end{array}\right),
$$

with

$$
-\omega^{2} z+A z+\mathrm{i} \omega B_{1} B_{1}^{*} z+\mathrm{i} \omega \mathrm{e}^{-\mathrm{i} \omega \tau} B_{2} B_{2}^{*} z=0 .
$$

It is an immediate consequence of the identity $(\mathrm{i} \omega I-\mathcal{A}) \varphi=0$.

First we notice that $\omega \neq 0$ since for $\omega=0$, the above identity reduces to $A z=0$ with $z \in D(A)$. Since by hypothesis $A$ is invertible, we get $z=0$ and therefore 0 is not an eigenvalue of $\mathcal{A}$.

We now take the duality bracket $\langle., .\rangle_{V^{\prime}, V}$ between (3.6) and $z \in V$ :

$$
\begin{aligned}
0 & =\left\langle-\omega^{2} z+A z+\mathrm{i} \omega B_{1} B_{1}^{*} z+\mathrm{i} \omega \mathrm{e}^{-\mathrm{i} \omega \tau} B_{2} B_{2}^{*} z, z\right\rangle_{V^{\prime}, V} \\
& =\left\langle\left(-\omega^{2} I+A\right) z, z\right\rangle_{V^{\prime}, V}+\mathrm{i} \omega\left\|B_{1}^{*} z\right\|_{U_{1}}^{2}+\mathrm{i} \omega \mathrm{e}^{-\mathrm{i} \omega \tau}\left\|B_{2}^{*} z\right\|_{U_{2}}^{2} .
\end{aligned}
$$

We look at the imaginary part of this expression to obtain

$$
\omega\left(\left\|B_{1}^{*} z\right\|_{U_{1}}^{2}+\cos (\omega \tau)\left\|B_{2}^{*} z\right\|_{U_{2}}^{2}\right)=0
$$

which implies, because $\omega \neq 0$,

We deduce that

$$
\left\|B_{1}^{*} z\right\|_{U_{1}}^{2}+\cos (\omega \tau)\left\|B_{2}^{*} z\right\|_{U_{2}}^{2}=0
$$

$$
0=\left\|B_{1}^{*} z\right\|_{U_{1}}^{2}+\cos (\omega \tau)\left\|B_{2}^{*} z\right\|_{U_{2}}^{2} \geq\left\|B_{1}^{*} z\right\|_{U_{1}}^{2}-\left\|B_{2}^{*} z\right\|_{U_{2}}^{2} \geq(1-\alpha)\left\|B_{1}^{*} z\right\|_{U_{1}}^{2} \geq 0,
$$

by (1.3) with $\alpha<1$. Consequently

which implies

$$
\left\|B_{1}^{*} z\right\|_{U_{1}}=0
$$

$$
B_{1}^{*} z=0 .
$$

Moreover, by (3.6), (3.7) and (1.3), we have

$$
A z=\omega^{2} z .
$$


Therefore, $z$ is an eigenvector of $A$ of associated eigenvalue $\omega^{2}$ such that

$$
B_{1}^{*} z=0,
$$

which contradicts (3.5). Thus, $\mathcal{A}$ has no eigenvalue on the imaginary axis.

Now, we can apply the main theorem of Arendt and Batty [5]: As $\sigma(\mathcal{A}) \cap \mathrm{i} \mathbb{R}$ is empty (because the surjectivity of $(\mathrm{i} \omega I-\mathcal{A})$ is equivalent to the injectivity of $\left.-\omega^{2}+A-\mathrm{i} \omega B_{1} B_{1}^{*}-\mathrm{i} \omega \mathrm{e}^{\mathrm{i} \omega \tau} B_{2} B_{2}^{*}\right)$, we obtain (3.4).

$\Rightarrow$ Let us show that (3.4) implies (3.5). For that purpose we use a contradiction argument. Suppose that there exists an eigenvector $\varphi$ of $A$ of associated eigenvalue $\lambda^{2}$ such that

$$
B_{1}^{*} \varphi=0 .
$$

Let us set

$$
\omega(., t)=\varphi \cos (\lambda t) .
$$

Then $\omega$ is solution of (1.2) and satisfies

$$
E(t)=E(0)
$$

because

and

$$
\left\|B_{1}^{*} \dot{\omega}(t)\right\|_{U_{1}}^{2}=\lambda^{2} \sin ^{2}(\lambda t)\left\|B_{1}^{*} \varphi\right\|_{U_{1}}^{2}=0
$$

$$
\begin{aligned}
\left\|B_{2}^{*} \dot{\omega}(t-\tau)\right\|_{U_{2}}^{2} & =\lambda^{2} \sin ^{2}(\lambda(t-\tau))\left\|B_{2}^{*} \varphi\right\|_{U_{2}}^{2} \\
& \leq \alpha \lambda^{2} \sin ^{2}(\lambda(t-\tau))\left\|B_{1}^{*} \varphi\right\|_{U_{1}}^{2}=0,
\end{aligned}
$$

by (1.3). This means that we have obtained a solution of system (1.2) with a constant energy, which contradicts (3.4).

\section{The EXPONENTIAL STABILITy}

\subsection{A priori estimate}

In order to obtain the characterization of decay properties of the damped system via observability inequalities for the conservative system we will use the following assumption from [3]:

If $\beta>0$ is fixed and $C_{\beta}=\{\lambda \in \mathbb{C} \mid \Re \lambda=\beta\}$, the function

$$
\lambda \in C_{\beta} \rightarrow H(\lambda)=\lambda B^{*}\left(\lambda^{2} I+A\right)^{-1} B \in \mathcal{L}(U) \text { is bounded, }
$$

where $B \in \mathcal{L}\left(U, V^{\prime}\right)$ with $U$ a Hilbert space.

We consider the evolution system

$$
\left\{\begin{array}{c}
\ddot{y}(t)+A y(t)=B v(t) \\
y(0)=\dot{y}(0)=0
\end{array}\right.
$$

and the following conservative system

$$
\left\{\begin{array}{c}
\ddot{\phi}(t)+A \phi(t)=0 \\
\phi(0)=\omega_{0}, \dot{\phi}(0)=\omega_{1} .
\end{array}\right.
$$

Let us recall the two following results proved in [3]:

Lemma 4.1. Suppose that $v \in L^{2}(0, T ; U)$ and that the solutions $\phi$ of (4.3) are such that $B^{*} \phi(.) \in H^{1}(0, T ; U)$ and there exists a constant $C>0$ such that

$$
\left\|\left(B^{*} \phi\right)^{\prime}(.)\right\|_{L^{2}(0, T ; U)} \leq C\left\|\left(\omega_{0}, \omega_{1}\right)\right\|_{V \times H} \quad \forall\left(\omega_{0}, \omega_{1}\right) \in V \times H .
$$


Then the system (4.2) admits a unique solution having the regularity

$$
y \in C(0, T ; V) \cap C^{1}(0, T ; H) .
$$

Proposition 4.2. Suppose that $v \in L^{2}(0, T ; U)$ and that the system (4.2) admits a unique solution having the regularity

$$
y \in C(0, T ; V) \cap C^{1}(0, T ; H) .
$$

Then hypothesis (4.1) holds if and only if $B^{*} y(.) \in H^{1}(0, T ; U)$ and there exists a constant $C>0$ independent of $T$ such that

$$
\left\|\left(B^{*} y\right)^{\prime}(.)\right\|_{L^{2}(0, T ; U)} \leq C \mathrm{e}^{\beta T}\|v\|_{L^{2}(0, T ; U)} .
$$

Let $\omega \in C(0, T ; V) \cap C^{1}(0, T ; H)$ be the solution of $(1.2)$ with $\left(\omega_{0}, \omega_{1}, f^{0}(-\tau .)\right)^{T} \in D(\mathcal{A})$. Then it can be split up in the form

$$
\omega=\phi+\psi
$$

where $\phi$ is solution of the system without damping (4.3), and $\psi$ satisfies

$$
\left\{\begin{array}{c}
\ddot{\psi}(t)+A \psi(t)=-B_{1} B_{1}^{*} \dot{\omega}(t)-B_{2} B_{2}^{*} \dot{\omega}(t-\tau) \\
\psi(0)=0, \dot{\psi}(0)=0
\end{array}\right.
$$

We now set $B=\left(B_{1} B_{2}\right) \in \mathcal{L}\left(U, V^{\prime}\right)$ where $U=U_{1} \times U_{2}$. It is easy to verify that $B^{*}=\left(\begin{array}{c}B_{1}^{*} \\ B_{2}^{*}\end{array}\right) \in \mathcal{L}(V, U)$. Therefore $\psi$ is solution of

$$
\left\{\begin{array}{c}
\ddot{\psi}(t)+A \psi(t)=B v(t) \\
\psi(0)=0, \dot{\psi}(0)=0
\end{array}\right.
$$

where $v(t)=\left(\begin{array}{c}-B_{1}^{*} \dot{\omega}(t) \\ -B_{2}^{*} \dot{\omega}(t-\tau)\end{array}\right)$. In other words, $\psi$ is solution of system (4.2) with

$$
B=\left(B_{1} B_{2}\right)
$$

and by Remark 3.2

$$
v=\left(\begin{array}{c}
-B_{1}^{*} \dot{\omega}(\cdot) \\
-B_{2}^{*} \dot{\omega}(\cdot-\tau)
\end{array}\right) \in L^{2}((0, T), U) .
$$

Then $\psi=\omega-\phi \in C(0, T ; V) \cap C^{1}(0, T ; H)$. Suppose that hypothesis (4.1) is satisfied for $B=\left(B_{1} B_{2}\right)$ and $U=U_{1} \times U_{2}$. By applying Proposition 4.2, we obtain

$$
\int_{0}^{T}\left\|\left(B^{*} \psi\right)^{\prime}\right\|_{U}^{2} \mathrm{~d} t \leq C \mathrm{e}^{2 \beta T} \int_{0}^{T}\|v(t)\|_{U}^{2} \mathrm{~d} t
$$

which is equivalent to

$$
\int_{0}^{T}\left(\left\|\left(B_{1}^{*} \psi\right)^{\prime}\right\|_{U_{1}}^{2}+\left\|\left(B_{2}^{*} \psi\right)^{\prime}\right\|_{U_{2}}^{2}\right) \mathrm{d} t \leq C \mathrm{e}^{2 \beta T} \int_{0}^{T}\left(\left\|B_{1}^{*} \dot{\omega}(t)\right\|_{U_{1}}^{2}+\left\|B_{2}^{*} \dot{\omega}(t-\tau)\right\|_{U_{2}}^{2}\right) \mathrm{d} t .
$$

In particular, we have

$$
\int_{0}^{T}\left\|\left(B_{1}^{*} \psi\right)^{\prime}\right\|_{U_{1}}^{2} \mathrm{~d} t \leq C \mathrm{e}^{2 \beta T} \int_{0}^{T}\left(\left\|B_{1}^{*} \dot{\omega}(t)\right\|_{U_{1}}^{2}+\left\|B_{2}^{*} \dot{\omega}(t-\tau)\right\|_{U_{2}}^{2}\right) \mathrm{d} t .
$$


Therefore, since $\omega=\phi+\psi$, we have

$$
\begin{aligned}
\int_{0}^{T}\left\|\left(B_{1}^{*} \phi\right)^{\prime}(t)\right\|_{U_{1}}^{2} \mathrm{~d} t & \leq 2\left(\int_{0}^{T}\left\|\left(B_{1}^{*} \omega\right)^{\prime}(t)\right\|_{U_{1}}^{2} \mathrm{~d} t+\int_{0}^{T}\left\|\left(B_{1}^{*} \psi\right)^{\prime}(t)\right\|_{U_{1}}^{2} \mathrm{~d} t\right) \\
& \leq C \mathrm{e}^{2 \beta T} \int_{0}^{T}\left(\left\|\left(B_{1}^{*} \dot{\omega}\right)(t)\right\|_{U_{1}}^{2}+\left\|\left(B_{2}^{*} \dot{\omega}\right)(t-\tau)\right\|_{U_{2}}^{2}\right) \mathrm{d} t .
\end{aligned}
$$

Thus, we have proved the following result:

Lemma 4.3. Suppose that assumption (4.1) is satisfied for $B=\left(B_{1} B_{2}\right), U=U_{1} \times U_{2}$. Then the solutions $\omega$ of (1.2) and $\phi$ of (4.3) satisfy

$$
\int_{0}^{T}\left\|\left(B_{1}^{*} \phi\right)^{\prime}(t)\right\|_{U_{1}}^{2} \mathrm{~d} t \leq C \mathrm{e}^{2 \beta T} \int_{0}^{T}\left(\left\|B_{1}^{*} \dot{\omega}(t)\right\|_{U_{1}}^{2}+\left\|B_{2}^{*} \dot{\omega}(t-\tau)\right\|_{U_{2}}^{2}\right) \mathrm{d} t
$$

with $C>0$ independent of $T$.

\subsection{The stability result}

Theorem 4.4. Assume that hypotheses (1.3) and (4.1) are verified for $B=\left(B_{1} B_{2}\right), U=U_{1} \times U_{2}$. If there exist a time $T>0$ and a constant $C>0$ such that the observability estimate

$$
\left\|A^{\frac{1}{2}} \omega_{0}\right\|_{H}^{2}+\left\|\omega_{1}\right\|_{H}^{2} \leq C \int_{0}^{T}\left\|\left(B_{1}^{*} \phi\right)^{\prime}(t)\right\|_{U_{1}}^{2} \mathrm{~d} t
$$

holds, where $\phi$ is solution of (4.3), then system (1.2) is exponentially stable in the energy space: there exist $C>0$ independent of $\tau$ and $\nu>0$ such that, for all initial data in $\mathcal{H}$,

$$
E(t) \leq C E(0) \mathrm{e}^{-\nu t} \quad \forall t>0 .
$$

Proof. Let $\omega$ be a solution of $(1.2)$ with initial data $\left(\omega_{0}, \omega_{1}, f^{0}(-\tau \cdot)\right) \in D(\mathcal{A})$.

Without loss of generality, we can always assume that (4.6) holds with $T>\tau$ and $C$ independent of $\tau$.

Integrating inequality (3.3) of Proposition 3.1 between 0 and $T$, we obtain

$$
\begin{aligned}
E(0)-E(T) & \geq C \int_{0}^{T}\left(\left\|B_{1}^{*} \dot{\omega}(t)\right\|_{U_{1}}^{2}+\left\|B_{2}^{*} \dot{\omega}(t-\tau)\right\|_{U_{2}}^{2}\right) \mathrm{d} t \\
& \geq \frac{C}{2} \int_{0}^{T}\left(\left\|B_{1}^{*} \dot{\omega}(t)\right\|_{U_{1}}^{2}+\left\|B_{2}^{*} \dot{\omega}(t-\tau)\right\|_{U_{2}}^{2}\right) \mathrm{d} t+\frac{C}{2} \int_{0}^{T}\left\|B_{2}^{*} \dot{\omega}(t-\tau)\right\|_{U_{2}}^{2} \mathrm{~d} t \\
& \geq C \mathrm{e}^{-2 \beta T}\left(\int_{0}^{T}\left\|\left(B_{1}^{*} \phi\right)^{\prime}(t)\right\|_{U_{1}}^{2} \mathrm{~d} t+\int_{0}^{T}\left\|B_{2}^{*} \dot{\omega}(t-\tau)\right\|_{U_{2}}^{2} \mathrm{~d} t\right) \text { by Lemma 4.3. }
\end{aligned}
$$

By assumption (4.6), we obtain

$$
E(0)-E(T) \geq C \mathrm{e}^{-2 \beta T}\left(\left\|A^{\frac{1}{2}} \omega_{0}\right\|_{H}^{2}+\left\|\omega_{1}\right\|_{H}^{2}+\int_{0}^{T}\left\|B_{2}^{*} \dot{\omega}(t-\tau)\right\|_{U_{2}}^{2} \mathrm{~d} t\right),
$$

with $C$ independent of $\tau$. As $T>\tau$, by change of variables, we have

$$
\begin{aligned}
\int_{0}^{T}\left\|B_{2}^{*} \dot{\omega}(t-\tau)\right\|_{U_{2}}^{2} \mathrm{~d} t & =\int_{-\tau}^{T-\tau}\left\|B_{2}^{*} \dot{\omega}(t)\right\|_{U_{2}}^{2} \mathrm{~d} t \\
& \geq \int_{-\tau}^{0}\left\|B_{2}^{*} \dot{\omega}(t)\right\|_{U_{2}}^{2} \mathrm{~d} t=\tau \int_{0}^{1}\left\|B_{2}^{*} \dot{\omega}(-\tau \rho)\right\|_{U_{2}}^{2} \mathrm{~d} \rho
\end{aligned}
$$


The two previous inequalities and (3.1) directly imply that

$$
E(0)-E(T) \geq C^{\prime} \mathrm{e}^{-2 \beta T}\left(\left\|A^{\frac{1}{2}} \omega_{0}\right\|_{H}^{2}+\left\|\omega_{1}\right\|_{H}^{2}+\xi \tau \int_{0}^{1}\left\|B_{2}^{*} \dot{\omega}(-\tau \rho)\right\|_{U_{2}}^{2} \mathrm{~d} \rho\right),
$$

with $C^{\prime}=C /(2 / \alpha-1)$. This means that for $T>\tau$, we have

$$
E(0)-E(T) \geq C^{\prime} \mathrm{e}^{-2 \beta T} E(0) .
$$

This estimate is equivalent to

$$
E(0)-E(T) \geq C^{\prime} \mathrm{e}^{-2 \beta T} E(T),
$$

because the energy is decreasing, which leads to

$$
E(T) \leq \gamma E(0)
$$

where $\gamma=\frac{1}{1+C^{\prime} \mathrm{e}^{-2 \beta T}}<1$. Applying this argument on $[(m-1) T, m T]$, for $m=1,2, \ldots$ (which is valid because the system is invariant by translation in time), we will get

$$
E(m T) \leq \gamma E((m-1) T) \leq \ldots \leq \gamma^{m} E(0) .
$$

Therefore, we have

$$
E(m T) \leq \mathrm{e}^{-\nu m T} E(0), m=1,2, \ldots
$$

with $\nu=\frac{1}{T} \ln \frac{1}{\gamma}=\frac{1}{T} \ln \left(1+C^{\prime} \mathrm{e}^{-2 \beta T}\right)>0$ which depends on $T$ and thus on $\tau$ (because $T>\tau$ ). For an arbitrary positive $t$, there exists $m \in \mathbb{N}^{*}$ such that $(m-1) T<t \leq m T$ and by the non-increasing property of the energy, we conclude

$$
E(t) \leq E((m-1) T) \leq \mathrm{e}^{-\nu(m-1) T} E(0) \leq \frac{1}{\gamma} \mathrm{e}^{-\nu t} E(0) .
$$

Hence the energy decays exponentially with the decay rate $\nu=\frac{1}{T} \ln \frac{1}{\gamma}=\frac{1}{T} \ln \left(1+C^{\prime} \mathrm{e}^{-2 \beta T}\right)<\frac{1}{\tau} \ln \left(1+C^{\prime} \mathrm{e}^{-2 \beta \tau}\right)$, because $T>\tau$. Notice that the constant $C$ of (4.7) can be chosen such that $C \geq 1+C^{\prime}$ (which is independent of $\tau$ ) because $\frac{1}{\gamma}=1+C^{\prime} \mathrm{e}^{-2 \beta T} \leq 1+C^{\prime}$.

By density of $D(\mathcal{A})$ into $\mathcal{H}$, we deduce that (4.7) holds for any initial data in $\mathcal{H}$.

Remark 4.5. In the previous theorem, we have seen that the decay rate is $\nu=\frac{1}{T} \ln \frac{1}{\gamma}=\frac{1}{T} \ln \left(1+C^{\prime} \mathrm{e}^{-2 \beta T}\right)<$ $\frac{1}{\tau} \ln \left(1+C^{\prime} \mathrm{e}^{-2 \beta \tau}\right)$ because $T>\tau$ and where $C^{\prime}$ is independent of $\tau$. Therefore, when $\tau$ becomes larger, the decay rate is slower.

Remark 4.6. Notice that the sufficient condition (4.6) for the exponential decay of the energy is the same than the case without delay (see [3]). Therefore, if the hypothesis (4.1) holds and if the dissipative system without delay (i.e. with $B_{2}=0$ ) is exponentially stable, then the system (1.2) is exponentially stable.

\section{The POLYNOMial STABILITY}

In some cases, the decay of the energy is not exponential, but can be polynomial. Our aim here is to give a sufficient condition that yields the explicit decay rate.

The proof of this stability result requires the next technical lemma proved in [2], Lemma 5.2.

Lemma 5.1. Let $\left(\varepsilon_{k}\right)_{k}$ be a sequence of positive real numbers satisfying

$$
\varepsilon_{k+1} \leq \varepsilon_{k}-C \varepsilon_{k+1}^{2+\mu} \quad \forall k \geq 0,
$$


where $C>0$ and $\mu>-1$ are constants. Then there exists a positive constant $M$ (depending on $\mu$ and $C$ ) such that

$$
\varepsilon_{k} \leq \frac{M}{(1+k)^{\frac{1}{1+\mu}}} \quad \forall k \geq 0
$$

with $M>\left(\frac{4}{(1+\mu) C}\right)^{\frac{1}{1+\mu}}$.

Moreover we recall the following interpolation result.

Lemma 5.2. For $\left(\omega_{0}, \omega_{1}\right) \in D(A) \times V$, we have

$$
\begin{aligned}
\left\|\omega_{0}\right\|_{D\left(A^{\frac{1}{2}}\right)}^{m+1} & \leq C\left\|\omega_{0}\right\|_{D(A)}^{m}\left\|\omega_{0}\right\|_{D\left(A^{\frac{1-m}{2}}\right)}, \\
\left\|\omega_{1}\right\|_{H}^{m+1} & \leq C\left\|\omega_{1}\right\|_{D\left(A^{\frac{1}{2}}\right)}^{m}\left\|\omega_{1}\right\|_{D\left(A^{-\frac{m}{2}}\right)},
\end{aligned}
$$

where $C>0$.

Proof. If we denote by $\left\{\lambda_{k_{n}}\right\}_{n}$ the eigenvalues of $A^{\frac{1}{2}}$ counted without multiplicity, $l_{n}$ the multiplicity of the eigenvalue $\lambda_{k_{n}}$ and $\left\{\varphi_{k_{n}+j}\right\}_{0 \leq j \leq l_{n}-1}$ the orthonormal eigenvectors associated with the eigenvalue $\lambda_{k_{n}}$, this lemma is a direct consequence of the equivalence $\|u\|_{D\left(A^{s}\right)}^{2} \sim \sum_{n \geq 1} \sum_{j=0}^{l_{n}-1}\left|u_{k_{n}+j}\right|^{2} \lambda_{k_{n}}^{4 s}$ for all $s \in \mathbb{R}$, when $u=\sum_{n \geq 1} \sum_{j=0}^{l_{n}-1} u_{k_{n}+j} \varphi_{k_{n}+j}$ and of Hölder's inequality with $p=1+\frac{1}{m}$ and $q=m+1$.

As for $\left(\omega_{0}, \omega_{1}, f^{0}(-\tau .)\right)^{\top} \in D(\mathcal{A}), \omega_{0}$ is not necessarily in $D(A)$, we can not apply Lemma 5.2 to $\omega_{0}$, and therefore we need to make the following hypothesis: there exists $C>0$ such that for all $\left(\omega_{0}, \omega_{1}, z\right)^{\top} \in D(\mathcal{A})$, we have

$$
\left\|\omega_{0}\right\|_{V}^{m+1} \leq C\left\|\left(\omega_{0}, \omega_{1}, z\right)\right\|_{D(\mathcal{A})}^{m}\left\|\omega_{0}\right\|_{D\left(A^{\frac{1-m}{2}}\right)} .
$$

Theorem 5.3. Let $\omega$ be a solution of $(1.2)$ with $\left(\omega_{0}, \omega_{1}, f^{0}(-\tau \cdot)\right)^{\top} \in D(\mathcal{A})$. Assume that the hypotheses (1.3), (4.1) and (5.2) are verified for $B=\left(B_{1} B_{2}\right), U=U_{1} \times U_{2}$. If there exist a positive real number $m$, a time $T>0$ and a constant $C>0$ such that

$$
\int_{0}^{T}\left\|\left(B_{1}^{*} \phi\right)^{\prime}(t)\right\|_{U_{1}}^{2} \mathrm{~d} t \geq C\left(\left\|\omega_{0}\right\|_{D\left(A^{\frac{1-m}{2}}\right)}^{2}+\left\|\omega_{1}\right\|_{D\left(A^{-\frac{m}{2}}\right)}^{2}\right)
$$

holds where $\phi$ is solution of (4.3), then the energy decays polynomially, i.e., there exists $C>0$ depending on $m$ and $\tau$ such that, for all initial data in $D(\mathcal{A})$,

$$
E(t) \leq \frac{C}{(1+t)^{\frac{1}{m}}}\left\|\left(\omega_{0}, \omega_{1}, f^{0}(-\tau \cdot)\right)\right\|_{D(\mathcal{A})}^{2} \quad \forall t>0 .
$$

Proof. As the hypothesis (4.1) is satisfied for $B=\left(B_{1} B_{2}\right), U=U_{1} \times U_{2}$, by using Lemma 4.3, we obtain

$$
\int_{0}^{T}\left(\left\|B_{1}^{*} \dot{\omega}(t)\right\|_{U_{1}}^{2}+\left\|B_{2}^{*} \dot{\omega}(t-\tau)\right\|_{U_{2}}^{2}\right) \mathrm{d} t \geq C \mathrm{e}^{-2 \beta T}\left(\left\|\omega_{0}\right\|_{D\left(A^{\frac{1-m}{2}}\right)}^{2}+\left\|\omega_{1}\right\|_{D\left(A^{-\frac{m}{2}}\right)}^{2}\right) .
$$


On the other hand, integrating the inequality (3.3) of Proposition 3.1 between 0 and $T^{*}$ for $T^{*}$ large enough: $T^{*} \geq \max (T, \tau)$, we have

$$
\begin{aligned}
E(0)-E\left(T^{*}\right) & \geq C \int_{0}^{T^{*}}\left(\left\|B_{1}^{*} \dot{\omega}(t)\right\|_{U_{1}}^{2}+\left\|B_{2}^{*} \dot{\omega}(t-\tau)\right\|_{U_{2}}^{2}\right) \mathrm{d} t \\
& \geq \frac{C}{2} \int_{0}^{T^{*}}\left(\left\|B_{1}^{*} \dot{\omega}(t)\right\|_{U_{1}}^{2}+\left\|B_{2}^{*} \dot{\omega}(t-\tau)\right\|_{U_{2}}^{2}\right) \mathrm{d} t+\frac{C}{2} \int_{0}^{T^{*}}\left\|B_{2}^{*} \dot{\omega}(t-\tau)\right\|_{U_{2}}^{2} \mathrm{~d} t \\
& \geq C \mathrm{e}^{-2 \beta T^{*}}\left(\left\|\omega_{0}\right\|_{D\left(A^{\frac{1-m}{2}}\right)}^{2}+\left\|\omega_{1}\right\|_{D\left(A^{-\frac{m}{2}}\right)}^{2}+\tau \int_{0}^{1}\left\|B_{2}^{*} \dot{\omega}(-\tau \rho)\right\|_{U_{2}}^{2} \mathrm{~d} \rho\right),
\end{aligned}
$$

by change of variable (because $T^{*}>\tau$ ). Therefore

$$
E\left(T^{*}\right) \leq E(0)-K_{1} \mathrm{e}^{-2 \beta T^{*}}\left(\left\|\omega_{0}\right\|_{D\left(A^{\frac{1-m}{2}}\right)}^{2}+\left\|\omega_{1}\right\|_{D\left(A^{-\frac{m}{2}}\right)}^{2}+\tau \xi \int_{0}^{1}\left\|B_{2}^{*} \dot{\omega}(-\tau \rho)\right\|_{U_{2}}^{2} \mathrm{~d} \rho\right),
$$

for some $K_{1}>0$ independent of $T^{*}$ and $\tau$.

Therefore, by (5.2), the previous interpolation result of Lemma 5.2 and a convexity inequality, we have:

$$
\begin{aligned}
\left\|\left(\omega_{0}, \omega_{1}\right)\right\|_{V \times H}^{m+1} & \leq C\left(\left\|\omega_{0}\right\|_{V}^{m+1}+\left\|\omega_{1}\right\|_{H}^{m+1}\right) \\
& \leq C\left(\left\|\left(\omega_{0}, \omega_{1}, f^{0}(-\tau .)\right)\right\|_{D(\mathcal{A})}^{m}\left\|\omega_{0}\right\|_{D\left(A^{\frac{1-m}{2}}\right)}+\left\|\omega_{1}\right\|_{D\left(A^{\frac{1}{2}}\right)}^{m}\left\|\omega_{1}\right\|_{D\left(A^{-\frac{m}{2}}\right)}\right) \\
& \leq C\left\|\left(\omega_{0}, \omega_{1}, f^{0}(-\tau .)\right)\right\|_{D(\mathcal{A})}^{m}\left(\left\|\omega_{0}\right\|_{D\left(A^{\frac{1-m}{2}}\right)}+\left\|\omega_{1}\right\|_{D\left(A^{-\frac{m}{2}}\right)}\right) .
\end{aligned}
$$

Denoting by $X_{-m}=D\left(A^{\frac{1-m}{2}}\right) \times D\left(A^{-\frac{m}{2}}\right)$, we have shown that

$$
\left\|\left(\omega_{0}, \omega_{1}\right)\right\|_{X_{-m}}^{2} \geq C \frac{\left\|\left(\omega_{0}, \omega_{1}\right)\right\|_{V \times H}^{2 m+2}}{\left\|\left(\omega_{0}, \omega_{1}, f^{0}(-\tau .)\right)\right\|_{D(\mathcal{A})}^{2 m}} .
$$

Now introduce the modified energy

$$
\tilde{E}(t)=\frac{1}{2}\|U(t)\|_{D(\mathcal{A})}^{2}=\frac{1}{2}\left(\|U(t)\|_{\mathcal{H}}^{2}+\|\mathcal{A} U(t)\|_{\mathcal{H}}^{2}\right) .
$$

As in Proposition 3.1, this energy $\tilde{E}$ is decaying.

Combining estimates (5.5) and (5.6), we obtain

$$
E\left(T^{*}\right) \leq E(0)-K_{2} \mathrm{e}^{-2 \beta T^{*}}\left(\frac{\left\|\left(\omega_{0}, \omega_{1}\right)\right\|_{V \times H}^{2 m+2}}{\tilde{E}(0)^{m}}+\xi \tau \int_{0}^{1}\left\|B_{2}^{*} \dot{\omega}(-\tau \rho)\right\|_{U_{2}}^{2} \mathrm{~d} \rho\right),
$$

for some $K_{2}>0$ independent of $T^{*}$ and $\tau$, or equivalently

$$
E\left(T^{*}\right) \leq E(0)-K_{2} \mathrm{e}^{-2 \beta T^{*}}\left(\frac{\left\|\left(\omega_{0}, \omega_{1}\right)\right\|_{V \times H}^{2 m+2}}{\tilde{E}(0)^{m}}+\xi \tau\left\|f^{0}(-\tau .)\right\|_{L^{2}\left((0,1), U_{2}\right)}^{2}\right) .
$$

Using the trivial estimate

$$
\begin{aligned}
(\xi \tau)^{m+1}\left\|f^{0}(-\tau .)\right\|_{L^{2}\left((0,1), U_{2}\right)}^{2 m+2} & =\xi \tau\left\|f^{0}(-\tau .)\right\|_{L^{2}\left((0,1), U_{2}\right)}^{2}(\xi \tau)^{m}\left\|f^{0}(-\tau .)\right\|_{L^{2}\left((0,1), U_{2}\right)}^{2 m} \\
& \leq \tau \xi\left\|f^{0}(-\tau \cdot)\right\|_{L^{2}\left((0,1), U_{2}\right)}^{2} \tilde{E}(0)^{m}
\end{aligned}
$$


the above inequality (5.7) becomes

$$
\begin{aligned}
E\left(T^{*}\right) & \leq E(0)-K_{2} \mathrm{e}^{-2 \beta T^{*}}\left(\frac{\left\|\left(\omega_{0}, \omega_{1}\right)\right\|_{V \times H}^{2 m+2}+(\xi \tau)^{m+1}\left\|f^{0}(-\tau .)\right\|_{L^{2}\left((0,1), U_{2}\right)}^{2 m+2}}{\tilde{E}(0)^{m}}\right) \\
& \leq E(0)-K^{\prime} \mathrm{e}^{-2 \beta T^{*}} \frac{E(0)^{m+1}}{\tilde{E}(0)^{m}}
\end{aligned}
$$

with $K^{\prime}>0$ independent of $T^{*}$ and $\tau$. Since the energy of our system is decaying, we obtain

$$
E\left(T^{*}\right) \leq E(0)-K^{\prime} \mathrm{e}^{-2 \beta T^{*}} \frac{E\left(T^{*}\right)^{m+1}}{\tilde{E}(0)^{m}}
$$

We now follow the method used in [2]. The estimate (5.8) being valid on the intervals $\left[k T^{*},(k+1) T^{*}\right]$, for any $k \geq 0$, we have

$$
E\left((k+1) T^{*}\right) \leq E\left(k T^{*}\right)-K^{\prime} \mathrm{e}^{-2 \beta T^{*}} \frac{E\left((k+1) T^{*}\right)^{m+1}}{\tilde{E}\left(k T^{*}\right)^{m}}
$$

Setting

$$
\varepsilon_{k}=\frac{E\left(k T^{*}\right)}{\tilde{E}(0)}
$$

and dividing (5.9) by $\tilde{E}(0)$, we obtain

$$
\varepsilon_{k+1} \leq \varepsilon_{k}-K^{\prime} \mathrm{e}^{-2 \beta T^{*}} \varepsilon_{k+1}^{m+1}
$$

because $\tilde{E}\left(k T^{*}\right) \leq \tilde{E}(0)$. By Lemma 5.1 with $\mu=m-1>-1$ (as $\left.m>0\right)$, there exists a constant $M^{\prime}>0$ (depending on $m$ and $K^{\prime} \mathrm{e}^{-2 \beta T^{*}}$, and verifying $M^{\prime}>\left(\frac{4 \mathrm{e}^{2 \beta T^{*}}}{m K^{\prime}}\right)^{\frac{1}{m}}$ ) such that

$$
\varepsilon_{k} \leq \frac{M^{\prime}}{(1+k)^{\frac{1}{m}}} \quad \forall k \geq 0
$$

or equivalently

$$
E\left(k T^{*}\right) \leq \frac{M^{\prime}}{(1+k)^{\frac{1}{m}}} \tilde{E}(0) .
$$

This estimate and again the decay of the energy lead to estimate (5.4), where $C=M^{\prime}\left(1+T^{*}\right)^{\frac{1}{m}}$.

Remark 5.4. Since the proof of the above theorem reveals that $C=M^{\prime}\left(1+T^{*}\right)^{\frac{1}{m}}$ with $M^{\prime}>\left(\frac{4 \mathrm{e}^{2 \beta T^{*}}}{m K^{\prime}}\right)^{\frac{1}{m}}$ and $T^{*}>\tau$, the constant $C$ depends on $\tau$ and when $\tau$ becomes larger, the decay rate becomes slower.

\section{Checking the observability inequalities}

In this section, we show how to obtain the observability inequalities used in Theorems 4.4 and 5.3. Our method is based on the generalized gap condition. Before giving spectral conditions to obtain exponential or polynomial decay, we recall some results about Ingham's inequality. 


\subsection{Preliminaries about Ingham's inequality}

Let $\left\{\lambda_{k}\right\}_{k \geq 1}$ be the set of eigenvalues of $A^{\frac{1}{2}}$ counted with their multiplicities (i.e. we repeat the eigenvalues according to their multiplicities). We further rewrite the sequence of eigenvalues $\left\{\lambda_{k}\right\}_{k \geq 1}$ as follows:

$$
\lambda_{k_{1}}<\lambda_{k_{2}}<\ldots<\lambda_{k_{i}}<\ldots
$$

where $k_{1}=1, k_{2}$ is the lowest index of the second distinct eigenvalue, $k_{3}$ is the lowest index of the third distinct eigenvalue, etc. For all $i \in \mathbb{N}^{*}$, let $l_{i}$ be the multiplicity of the eigenvalue $\lambda_{k_{i}}$, i.e.

$$
\lambda_{k_{i}-1}<\lambda_{k_{i}}=\lambda_{k_{i}+1}=\ldots=\lambda_{k_{i}+l_{i}-1}<\lambda_{k_{i}+l_{i}}=\lambda_{k_{i+1}} .
$$

We have $k_{1}=1, k_{2}=l_{1}, k_{3}=l_{1}+l_{2}$, etc. Let $\left\{\varphi_{k_{i}+j}\right\}_{0 \leq j \leq l_{i}-1}$ be the orthonormal eigenvectors associated with the eigenvalue $\lambda_{k_{i}}$. We assume that the following generalized gap condition holds:

$$
\exists M \in \mathbb{N}^{*}, \exists \gamma_{0}>0, \forall k \geq 1, \lambda_{k+M}-\lambda_{k} \geq M \gamma_{0}
$$

Fix a positive real number $\gamma_{0}^{\prime} \leq \gamma_{0}$ and denote by $A_{k}, k=1, \ldots, M$ the set of natural numbers $k_{m}$ satisfying (see for instance [6])

$$
\left\{\begin{array}{l}
\lambda_{k_{m}}-\lambda_{k_{m-1}} \geq \gamma_{0}^{\prime} \\
\lambda_{k_{n}}-\lambda_{k_{n-1}}<\gamma_{0}^{\prime} \\
\lambda_{k_{m+k}}-\lambda_{k_{m+k-1}} \geq \gamma_{0}^{\prime} .
\end{array} \quad \text { for } m+1 \leq n \leq m+k-1,\right.
$$

Then one easily checks that the sets $A_{k}+j, j=0, \ldots, k-1, k=1, \ldots, M$ form a partition of $\mathbb{N}^{*}$. Notice that some sets $A_{k}$ may be empty because, for the generalized gap condition, the choice of $M$ takes into account multiple eigenvalues.

Now for $k_{m} \in A_{k}$, we recall that the finite differences $e_{m+j}(t), j=0, \ldots, k-1$, corresponding to the exponential functions $\mathrm{e}^{-\mathrm{i} \lambda_{k_{m+j}} t}, j=0, \ldots, k-1$ are given by

$$
e_{m+j}(t)=\sum_{p=m}^{m+j} \prod_{\substack{q=m \\ q \neq p}}^{m+j}\left(\lambda_{k_{p}}-\lambda_{k_{q}}\right)^{-1} \mathrm{e}^{\mathrm{i} \lambda_{k_{p}} t} .
$$

Write for shortness, $e_{-n}(t)$ the same finite differences functions corresponding to $-\lambda_{k_{n}}$.

Now we are ready to recall the next inequality of Ingham's type, see for instance Theorem 1.5 of [6]:

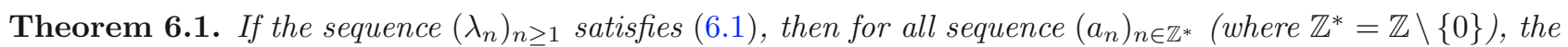
function

$$
f(t)=\sum_{n \in \mathbb{Z}^{*}} a_{n} e_{n}(t)
$$

satisfies the estimates

$$
\int_{0}^{T}|f(t)|^{2} \mathrm{~d} t \sim \sum_{n \in \mathbb{Z}^{*}}\left|a_{n}\right|^{2}
$$

for $T>\frac{2 \pi}{\gamma_{0}}$.

Going back to the original functions $\mathrm{e}^{\mathrm{i} \lambda_{k_{n}} t}$, the above equivalence (6.2) means that, for $T>\frac{2 \pi}{\gamma_{0}}$, the function (from now on $\lambda_{-k_{n}}=-\lambda_{k_{n}}$ )

$$
f(t)=\sum_{n \in \mathbb{Z}^{*}} \alpha_{n} \mathrm{e}^{\mathrm{i} \lambda_{k_{n}} t},
$$


satisfies the estimates

$$
\int_{0}^{T}|f(t)|^{2} \mathrm{~d} t \sim \sum_{k=1}^{M} \sum_{\left|k_{n}\right| \in A_{k}}\left\|B_{k_{n}}^{-1} C_{k_{n}}\right\|_{2}^{2},
$$

where $\|\cdot\|_{2}$ means the Euclidean norm of the vector, for $k_{n} \in A_{k}$ the vector $C_{k_{n}}$ is given by

$$
C_{k_{n}}=\left(\alpha_{n}, \ldots, \alpha_{n+k-1}\right)^{T}
$$

and the $k \times k$ matrix $B_{k_{n}}$ allows to pass from the coefficients $a_{k_{n}}$ to $\alpha_{k_{n}}$, namely

$$
C_{k_{n}}=B_{k_{n}} \cdot\left(a_{n}, \ldots, a_{n+k-1}\right)^{T}
$$

and is given by $B_{k_{n}}=\left(B_{k_{n}, i j}\right)_{1 \leq i, j \leq k}$ the matrix of size $k \times k$ such that

$$
B_{k_{n}, i j}= \begin{cases}\prod_{\substack{q=n \\ q \neq n+i-1}}^{n+j-1}\left(\lambda_{k_{n+i-1}}-\lambda_{k_{q}}\right)^{-1} & \text { if } i \leq j,(i, j) \neq(1,1), \\ 1 & \text { if }(i, j)=(1,1) \\ 0 & \text { if } i>j .\end{cases}
$$

More explicitly, we have

$$
B_{k_{n}}=\left(\begin{array}{ccccc}
1 & \frac{1}{\lambda_{k_{n}}-\lambda_{k_{n+1}}} & \frac{1}{\left(\lambda_{k_{n}}-\lambda_{k_{n}+1}\right)\left(\lambda_{k_{n}}-\lambda_{k_{n+2}}\right)} & \cdots & \frac{1}{\left(\lambda_{k_{n}}-\lambda_{k_{n+1}}\right) \cdots\left(\lambda_{k_{n}}-\lambda_{k_{n+k-1}}\right)} \\
0 & \frac{1}{\lambda_{k_{n+1}}-\lambda_{k_{n}}} & \frac{1}{\left(\lambda_{k_{n+1}}-\lambda_{k_{n}}\right)\left(\lambda_{k_{n+1}}-\lambda_{k_{n+2}}\right)} & \cdots & \frac{1}{\left(\lambda_{k_{n+1}}-\lambda_{k_{n}}\right) \cdots\left(\lambda_{k_{n+1}}-\lambda_{k_{n+k-1}}\right)} \\
0 & 0 & \frac{1}{\left(\lambda_{k_{n+2}}-\lambda_{k_{n}}\right)\left(\lambda_{k_{n+2}}-\lambda_{k_{n+1}}\right)} & \cdots & \frac{1}{\left(\lambda_{k_{n+2}}-\lambda_{k_{n}}\right) \cdots\left(\lambda_{k_{n+2}}-\lambda_{k_{n+k-1}}\right)} \\
\vdots & \vdots & 0 & \ddots & \vdots \\
0 & 0 & & \cdots & \frac{1}{\left(\lambda_{k_{n+k-1}}-\lambda_{k_{n}}\right) \cdots\left(\lambda_{k_{n+k-1}}-\lambda_{k_{n+k-2}}\right)}
\end{array}\right)
$$

We proceed similarly for $n \leq-1$, the indices being decreasing from $n$ to $n-k+1$.

Remark 6.2. If the standard gap condition

$$
\exists \gamma_{0}>0, \forall n \geq 1, \lambda_{k_{n+1}}-\lambda_{k_{n}} \geq \gamma_{0}
$$

holds, then $A_{1}=\mathbb{Z}^{*}$ and $B_{1}=1$ and in that case $f(t)=\sum_{n \in \mathbb{Z}^{*}} \alpha_{n} \mathrm{e}^{\mathrm{i} \lambda_{k_{n}} t}$ satisfies Ingham's inequality (see [13]):

$$
\int_{0}^{T}|f(t)|^{2} \mathrm{~d} t \sim \sum_{n \in \mathbb{Z}^{*}}\left|\alpha_{n}\right|^{2}
$$

for $T>\frac{2 \pi}{\gamma_{0}}$.

Now, let $U$ be a separable Hilbert space (in the sequel, $U$ will be $U_{1}$ ). For a vector $c=\left(\begin{array}{c}c_{1} \\ \vdots \\ c_{m}\end{array}\right)$ in $U^{m}$, we set $\|\cdot\|_{U, 2}$ the norm in $U^{m}$ defined by

$$
\|c\|_{U, 2}^{2}=\sum_{l=1}^{m}\left\|c_{l}\right\|_{U}^{2}
$$

Then we obtain the inequality of Ingham's type in $U$ : 
Proposition 6.3. If we have the standard gap condition (6.4), then for all sequence $\left(a_{n}\right)_{n}$ in $U$, the function

$$
u(t)=\sum_{n \in \mathbb{Z}^{*}} a_{n} \mathrm{e}^{\mathrm{i} \lambda_{k_{n}} t}
$$

satisfies the estimates

$$
\int_{0}^{T}\|u(t)\|_{U}^{2} \mathrm{~d} t \sim \sum_{n \in \mathbb{Z}^{*}}\left\|a_{n}\right\|_{U}^{2}
$$

for $T>\frac{2 \pi}{\gamma_{0}}$.

Proof. As $U$ is a separable Hilbert space, there exists a Hilbert basis $\left(\psi_{k}\right)_{k \geq 1}$ of $U$. Therefore, $a_{n} \in U$ can be written as

$$
a_{n}=\sum_{k=1}^{+\infty} a_{n}^{k} \psi_{k}
$$

We truncate $a_{n}$ as follows: for $K \in \mathbb{N}^{*}$, let $a_{n}^{(K)}=\sum_{k=1}^{K} a_{n}^{k} \psi_{k}$ and set $u_{K}(t)=\sum_{k=1}^{K}\left(\sum_{n \in \mathbb{Z}^{*}} a_{n}^{k} \mathrm{e}^{\mathrm{i} \lambda_{k_{n}} t}\right) \psi_{k}$. Since $\left(\psi_{k}\right)_{k \geq 1}$ is a Hilbert basis, we have by Fubini's theorem

$$
\left\|u_{K}(t)\right\|_{U}^{2}=\sum_{k=1}^{K}\left|\sum_{n \in \mathbb{Z}^{*}} a_{n}^{k} \mathrm{e}^{\mathrm{i} \lambda_{k_{n}} t}\right|^{2} .
$$

Thus, by applying Ingham's inequality (6.5), we have

$$
\begin{aligned}
\int_{0}^{T}\left\|u_{K}(t)\right\|_{U}^{2} \mathrm{~d} t & =\sum_{k=1}^{K} \int_{0}^{T}\left|\sum_{n \in \mathbb{Z}^{*}} a_{n}^{k} \mathrm{e}^{\mathrm{i} \lambda_{k_{n}} t}\right|^{2} \mathrm{~d} t \\
& \sim \sum_{k=1}^{K} \sum_{n \in \mathbb{Z}^{*}}\left(a_{n}^{k}\right)^{2} \\
& \sim \sum_{n \in \mathbb{Z}^{*} k=1} \sum_{k=1}^{K}\left(a_{n}^{k}\right)^{2} .
\end{aligned}
$$

Therefore

$$
\int_{0}^{T}\left\|u_{K}(t)\right\|_{U}^{2} \mathrm{~d} t \sim \sum_{n \in \mathbb{Z}^{*}}\left\|a_{n}^{(K)}\right\|_{U}^{2}
$$

As $u_{K} \rightarrow u$ and $a_{n}^{(K)} \rightarrow a_{n}$ when $K \rightarrow+\infty$, we obtain the result.

In the same way, we obtain an Ingham's type inequality in a Hilbert space $U$ in the case of the generalized gap condition (6.1).

Corollary 6.4. If the sequence $\left(\lambda_{n}\right)_{n \geq 1}$ satisfies (6.1), then for all sequence $\left(\alpha_{n}\right)_{n \in \mathbb{Z}^{*}}$ in $U$, the function

$$
f(t)=\sum_{n \in \mathbb{Z}^{*}} \alpha_{n} \mathrm{e}^{\mathrm{i} \lambda_{k_{n}} t},
$$

satisfies the estimates

$$
\int_{0}^{T}|f(t)|^{2} \mathrm{~d} t \sim \sum_{k=1}^{M} \sum_{\left|k_{n}\right| \in A_{k}}\left\|B_{k_{n}}^{-1} C_{k_{n}}\right\|_{U, 2}^{2}
$$


for $T>\frac{2 \pi}{\gamma_{0}}$, where

$$
C_{k_{n}}=\left(\alpha_{n}, \ldots, \alpha_{n+k-1}\right)^{T} \in U^{k}
$$

\subsection{A first observability inequality}

Proposition 6.5. Assume that the generalized gap condition (6.1) holds and that $U_{1}$ is separable. Let $\phi$ be the solution of (4.3) with $\left(\omega_{0}, \omega_{1}\right) \in V \times H$. Then there exists a time $T>0$ and a constant $C>0$ (depending on $T$ ) such that (4.6) holds if and only if

$$
\exists \gamma>0, \forall k=1, \ldots, M, \forall k_{n} \in A_{k}, \forall \xi \in \mathbb{R}^{L_{n}},\left\|B_{k_{n}}^{-1} \Phi_{k_{n}} \xi\right\|_{U_{1}, 2} \geq \gamma\|\xi\|_{2},
$$

where the matrix $\Phi_{k_{n}}$ with coefficients in $U_{1}$ and size $k \times L_{n}$, where $L_{n}=\sum_{i=1}^{k} l_{n+i-1}-1$, is given as follow: for all $i=1, \ldots, k$, we set

$$
\left(\Phi_{k_{n}}\right)_{i j}= \begin{cases}B_{1}^{*} \varphi_{k_{n+i-1}+j-L_{n, i-1}} & \text { if } L_{n, i-1}<j \leq L_{n, i} \\ 0 & \text { else }\end{cases}
$$

where

$$
\begin{gathered}
L_{n, 0}=0, \\
L_{n, i}=\sum_{i^{\prime}=1}^{i} l_{n+i^{\prime}-1}-1 \quad \forall i \geq 1 .
\end{gathered}
$$

Proof. We first show that $(6.7) \Rightarrow(4.6)$. Writing

$$
\omega_{0}=\sum_{i \geq 1} \sum_{j=0}^{l_{i}-1} a_{k_{i}+j} \varphi_{k_{i}+j}
$$

and

$$
\omega_{1}=\sum_{i \geq 1} \sum_{j=0}^{l_{i}-1} b_{k_{i}+j} \varphi_{k_{i}+j}
$$

where $\left(\lambda_{k_{i}} a_{k_{i}+j}\right)_{i, j},\left(b_{k_{i}+j}\right)_{i, j} \in l^{2}\left(\mathbb{N}^{*}\right)$, then the solution $\phi$ of system without damping (4.3) is given by

$$
\phi(\cdot, t)=\sum_{i \geq 1} \sum_{j=0}^{l_{i}-1}\left(a_{k_{i}+j} \cos \left(\lambda_{k_{i}} t\right)+\frac{b_{k_{i}+j}}{\lambda_{k_{i}}} \sin \left(\lambda_{k_{i}} t\right)\right) \varphi_{k_{i}+j} .
$$

Consequently

$$
\left(B_{1}^{*} \phi\right)^{\prime}(t)=\sum_{i \geq 1} \sum_{j=0}^{l_{i}-1}\left(-a_{k_{i}+j} \lambda_{k_{i}} \sin \left(\lambda_{k_{i}} t\right)+b_{k_{i}+j} \cos \left(\lambda_{k_{i}} t\right)\right) B_{1}^{*} \varphi_{k_{i}+j} .
$$

By grouping the terms corresponding to the same eigenvalue, we get

$$
\begin{aligned}
\left(B_{1}^{*} \phi\right)^{\prime}(t) & =\sum_{i \geq 1}\left(\sum_{j=0}^{l_{i}-1}-a_{k_{i}+j} B_{1}^{*} \varphi_{k_{i}+j}\right) \lambda_{k_{i}} \sin \left(\lambda_{k_{i}} t\right)+\sum_{i \geq 1}\left(\sum_{j=0}^{l_{j}-1} b_{k_{i}+j} B_{1}^{*} \varphi_{k_{i}+j}\right) \cos \left(\lambda_{k_{i}} t\right) \\
& =\sum_{n \in \mathbb{Z}^{*}} \alpha_{n} \mathrm{e}^{\mathrm{i} \lambda_{k_{n}} t}
\end{aligned}
$$


where

$$
\begin{gathered}
\alpha_{n}=\frac{1}{2}\left(\left(\sum_{j=0}^{l_{n}-1} b_{k_{n}+j} B_{1}^{*} \varphi_{k_{n}+j}\right)+\mathrm{i}\left(\sum_{j=0}^{l_{n}-1} a_{k_{n}+j} B_{1}^{*} \varphi_{k_{n}+j}\right) \lambda_{k_{n}}\right) \quad \forall n \geq 1, \\
\alpha_{-n}=\frac{1}{2}\left(\left(\sum_{j=0}^{l_{n}-1} b_{k_{n}+j} B_{1}^{*} \varphi_{k_{n}+j}\right)-\mathrm{i}\left(\sum_{j=0}^{l_{n}-1} a_{k_{n}+j} B_{1}^{*} \varphi_{k_{n}+j}\right) \lambda_{k_{n}}\right) \quad \forall n \geq 1 .
\end{gathered}
$$

Integrating the square of the norm of this identity between 0 and $T>0$ and using Ingham's inequality (6.6) in $U_{1}$, for $T$ large enough, we get

$$
\int_{0}^{T}\left\|\left(B_{1}^{*} \phi\right)^{\prime}(t)\right\|_{U_{1}}^{2} \mathrm{~d} t \geq C \sum_{k=1}^{M} \sum_{\left|k_{n}\right| \in A_{k}}\left\|B_{k_{n}}^{-1} C_{k_{n}}\right\|_{U_{1}, 2}^{2},
$$

where $C_{k_{n}}=\left(\alpha_{n}, \ldots, \alpha_{n+k-1}\right)^{T}$ is a vector of $U_{1}^{k}$.

But for all $k_{n} \in A_{k}$, setting

$$
\begin{gathered}
\tilde{A}_{k_{n}}=\left(\lambda_{k_{n}} a_{k_{n}}, \ldots, \lambda_{k_{n}} a_{k_{n}+l_{n}-1}, \lambda_{k_{n+1}} a_{k_{n+1}}, \ldots, \lambda_{k_{n+1}} a_{k_{n+1}+l_{n+1}-1}, \ldots,\right. \\
\left.\lambda_{k_{n+k-1}} a_{k_{n+k-1}}, \ldots, \lambda_{k_{n+k-1}} a_{k_{n+k-1}+l_{n+k-1}-1}\right)^{T} \\
\tilde{B}_{k_{n}}=\left(b_{k_{n}}, \ldots, b_{k_{n}+l_{n}-1}, b_{k_{n+1}}, \ldots, b_{k_{n+1}+l_{n+1}-1}, \ldots, b_{k_{n+k-1}}, \ldots\right. \\
\left.b_{k_{n+k-1}+l_{n+k-1}-1}\right)^{T}
\end{gathered}
$$

we readily check that

$$
\int_{0}^{T}\left\|\left(B_{1}^{*} \phi\right)^{\prime}(t)\right\|_{U_{1}}^{2} \mathrm{~d} t \geq C \sum_{k=1}^{M} \sum_{\left|k_{n}\right| \in A_{k}}\left(\left\|B_{k_{n}}^{-1} \Phi_{k_{n}} \tilde{A}_{k_{n}}\right\|_{U_{1}, 2}^{2}+\left\|B_{k_{n}}^{-1} \Phi_{k_{n}} \tilde{B}_{k_{n}}\right\|_{U_{1}, 2}^{2}\right) .
$$

Hence the assumption (6.7) yields

$$
\begin{aligned}
\int_{0}^{T}\left\|\left(B_{1}^{*} \phi\right)^{\prime}(t)\right\|_{U_{1}}^{2} \mathrm{~d} t & \geq C \sum_{k=1\left|k_{n}\right| \in A_{k}}^{M}\left(\left\|\tilde{A}_{k_{n}}\right\|_{2}^{2}+\left\|\tilde{B}_{k_{n}}\right\|_{2}^{2}\right) \\
& =C\left(\left\|A^{\frac{1}{2}} \omega_{0}\right\|_{H}^{2}+\left\|\omega_{1}\right\|_{H}^{2}\right)
\end{aligned}
$$

because $\left(\varphi_{k_{n}+i}\right)_{n, i}$ is an orthonormal basis associated with the operator $A^{\frac{1}{2}}$.

It remains to show that $(4.6) \Rightarrow(6.7)$.

Let $k=1, \ldots, M$ and $k_{n} \in A_{k}$ be fixed. Take $\omega_{0}=\sum_{i=n}^{n+k-1} \sum_{j=0}^{l_{i}-1} a_{k_{i}+j} \varphi_{k_{i}+j}$ and $\omega_{1}=$ $\sum_{i=n}^{n+k-1} \sum_{j=0}^{l_{i}-1} b_{k_{i}+j} \varphi_{k_{i}+j}$. Then the solution $\phi$ of system (4.3) is given by

$$
\phi(\cdot, t)=\sum_{i=n}^{n+k-1} \sum_{j=0}^{l_{i}-1}\left(a_{k_{i}+j} \cos \left(\lambda_{k_{i}} t\right)+\frac{b_{k_{i}+j}}{\lambda_{k_{i}}} \sin \left(\lambda_{k_{i}} t\right)\right) \varphi_{k_{i}+j}
$$

and then

$$
\left(B_{1}^{*} \phi\right)^{\prime}(t)=\sum_{i=n}^{n+k-1} \sum_{j=0}^{l_{i}-1}\left(-a_{k_{i}+j} \lambda_{k_{i}} \sin \left(\lambda_{k_{i}} t\right)+b_{k_{i}+j} \cos \left(\lambda_{k_{i}} t\right)\right) B_{1}^{*} \varphi_{k_{i}+j} .
$$


Applying again Ingham's inequality, we get for $T$ large enough and $\tilde{A}_{k_{n}}, \tilde{B}_{k_{n}}$ defined above

$$
\int_{0}^{T}\left\|\left(B_{1}^{*} \phi\right)^{\prime}(t)\right\|_{U_{1}}^{2} \mathrm{~d} t \sim\left\|B_{k_{n}}^{-1} \Phi_{k_{n}} \tilde{A}_{k_{n}}\right\|_{U_{1}, 2}^{2}+\left\|B_{k_{n}}^{-1} \Phi_{k_{n}} \tilde{B}_{k_{n}}\right\|_{U_{1}, 2}^{2} .
$$

By (4.6), we obtain

$$
\begin{aligned}
\left\|B_{k_{n}}^{-1} \Phi_{k_{n}} \tilde{A}_{k_{n}}\right\|_{U_{1}, 2}^{2}+\left\|B_{k_{n}}^{-1} \Phi_{k_{n}} \tilde{B}_{k_{n}}\right\|_{U_{1}, 2}^{2} & \geq C\left\|A^{\frac{1}{2}} \omega_{0}\right\|_{H}^{2}+\left\|\omega_{1}\right\|_{H}^{2} \\
& =C \sum_{i=n}^{n+k-1} \sum_{j=0}^{n+1}\left(a_{k_{i}+j}^{2} \lambda_{k_{i}}^{2}+b_{k_{i}+j}^{2}\right),
\end{aligned}
$$

for some $C>0$. Hence we conclude that

$$
\left\|B_{k_{n}}^{-1} \Phi_{k_{n}} \xi\right\|_{U_{1}, 2} \geq \gamma\|\xi\|_{2}
$$

This ends the proof.

Remark 6.6. If the standard gap condition (6.4) holds, then $A_{1}=\mathbb{N}^{*}$ and $B_{1}=1$. In this case, the assumption (6.7) becomes

$$
\exists \gamma>0, \forall k_{n} \geq 1, \forall \xi \in \mathbb{R}^{l_{n}},\left\|\Phi_{k_{n}} \xi\right\|_{U_{1}} \geq \gamma\|\xi\|_{2} .
$$

Moreover, if the standard gap condition (6.4) holds and if the eigenvalues are simple, the assumption (6.7) becomes

$$
\exists \gamma>0, \forall k \geq 1,\left\|B_{1}^{*} \varphi_{k}\right\|_{U_{1}} \geq \gamma
$$

Remark 6.7. The above Proposition 6.5 yields a time $T>0$ and a constant $C>0$ depending on $T$ such that (4.6) holds but the time $T$ and the constant $C$ do not depend on the delay $\tau$. Hence if the minimal time $T$ is not strictly greater than $\tau$, by choosing $T^{\prime}>\max \{T, \tau\}$, we still have

$$
\left\|A^{\frac{1}{2}} \omega_{0}\right\|_{H}^{2}+\left\|\omega_{1}\right\|_{H}^{2} \leq C \int_{0}^{T^{\prime}}\left\|\left(B_{1}^{*} \phi\right)^{\prime}(t)\right\|_{U_{1}}^{2} \mathrm{~d} t
$$

with the same constant $C$ as before and that does not depend on $\tau$. This means that under the generalized gap condition (6.1), the condition (6.7) is equivalent to the sufficient condition of Theorem 4.4.

\subsection{A second observability inequality}

Proposition 6.8. Assume that the generalized gap condition (6.1) holds and that $U_{1}$ is separable. Let $\phi$ be the solution of (4.3) with $\left(\omega_{0}, \omega_{1}\right) \in V \times H$. Then for a fixed real number $m>0$, there exist a time $T>0$ and a constant $C>0$ such that (5.3) holds if and only if

$$
\exists \gamma>0, \forall k=1, \ldots, M, \forall k_{n} \in A_{k}, \forall \xi \in \mathbb{R}^{L_{n}},\left\|B_{k_{n}}^{-1} \Phi_{k_{n}} \xi\right\|_{U_{1}, 2} \geq \frac{\gamma}{\lambda_{k_{n}}^{m}}\|\xi\|_{2} .
$$

Proof. The proof is similar to the one of Proposition 6.5 because

$$
\begin{aligned}
\sum_{n \geq 1} \frac{1}{\lambda_{k_{n}}^{2 m}} \sum_{j=0}^{l_{n}-1}\left(a_{k_{n}+j}^{2} \lambda_{k_{n}}^{2}+b_{k_{n}+j}^{2}\right) & =\sum_{n \geq 1} \sum_{j=0}^{l_{n}-1}\left(a_{k_{n}+j}^{2} \lambda_{k_{n}}^{2(1-m)}+b_{k_{n}+j}^{2} \lambda_{k_{n}}^{-2 m}\right) \\
& \sim\left\|\omega_{0}\right\|_{D\left(A^{\frac{1-m}{2}}\right)}^{2}+\left\|\omega_{1}\right\|_{D\left(A^{-\frac{m}{2}}\right)}^{2} .
\end{aligned}
$$

The details are therefore omitted. 
Remark 6.9. If the standard gap condition (6.4) holds, the assumption (6.10) becomes

$$
\exists \gamma>0, \forall k_{n} \geq 1, \forall \xi \in \mathbb{R}^{l_{n}},\left\|\Phi_{k_{n}} \xi\right\|_{U_{1}} \geq \frac{\gamma}{\lambda_{k_{n}}^{m}}\|\xi\|_{2} .
$$

Moreover, if the standard gap condition (6.4) holds and if the eigenvalues are simple, the assumption (6.10) becomes

$$
\exists \gamma>0, \forall k \geq 1,\left\|B_{1}^{*} \varphi_{k}\right\|_{U_{1}} \geq \frac{\gamma}{\lambda_{k_{n}}^{m}}
$$

A remark similar to Remark 6.7 can be made for polynomial stability.

\section{EXAmples}

We end up this paper by considering different examples for which our abstract framework can be applied. To our knowledge, all the examples, with the exception of the first one, are new.

\subsection{A wave equation on 1-d networks with nodal feedbacks}

In this section we show that the result obtained in [17] enter in the framework of this paper. Obviously we will use the same notations for a network that in [17] that we briefly recall (for more details see [17]). We denote by $\mathcal{E}=\left\{e_{j} ; 1 \leq j \leq N\right\}$ the set of edges $e_{j}$ of length $l_{j}>0$ of a given network $\mathcal{R}$ and $\mathcal{V}$ the set of vertices of $\mathcal{R}$. For a function $u: \mathcal{R} \rightarrow \mathbb{R}$, we set $u_{j}=u_{\mid e_{j}}$ the restriction of $u$ to $e_{j}$. For a fixed vertex $v$, we set

$$
\mathcal{E}_{v}=\left\{j \in\{1, \ldots, N\} ; v \in \overline{e_{j}}\right\} .
$$

If $\operatorname{card}\left(\mathcal{E}_{v}\right) \geq 2, v$ is an interior node. Let $\mathcal{V}_{\text {int }}$ be the set of interior nodes. If $\operatorname{card}\left(\mathcal{E}_{v}\right)=1, v$ is an exterior node. Let $\mathcal{V}_{\text {ext }}$ be the set of exterior nodes. For $v \in \mathcal{V}_{\text {ext }}$, we set $\mathcal{E}_{v}=\left\{j_{v}\right\}$.

We now fix a partition of $\mathcal{V}_{\text {ext }}$ :

$$
\mathcal{V}_{\text {ext }}=\mathcal{D} \cup \mathcal{N} \cup \mathcal{V}_{\text {ext }}^{c}, \text { where } \mathcal{D} \neq \emptyset
$$

We actually will impose Dirichlet boundary condition at the nodes of $\mathcal{D}$, Neumann boundary condition at the nodes of $\mathcal{N}$ and finally a feedback boundary condition at the nodes of $\mathcal{V}_{\text {ext }}^{c}$. We further fix a subset $\mathcal{V}_{\text {int }}^{c}$ of $\mathcal{V}_{\text {int }}$ where a feedback transmission condition will be imposed. By shortness, we denote by $\mathcal{V}_{c}$ the set of controlled nodes, namely

$$
\mathcal{V}_{c}=\mathcal{V}_{\text {int }}^{c} \cup \mathcal{V}_{\text {ext }}^{c}
$$

We here consider the following initial and boundary system:

$$
\begin{cases}\frac{\partial^{2} u_{j}}{\partial t^{2}}(x, t)-\frac{\partial^{2} u_{j}}{\partial x^{2}}(x, t)=0 & 0<x<l_{j}, t>0, \forall j \in\{1, \ldots, N\} \\ u_{j}(v, t)=u_{l}(v, t)=u(v, t) & t>0, \forall j, l \in \mathcal{E}_{v}, v \in \mathcal{V}_{\mathrm{int}} \\ \sum_{j \in \mathcal{E}_{v}} \frac{\partial u_{j}}{\partial n_{j}}(v, t)=-\alpha_{1}^{(v)} \frac{\partial u}{\partial t}(v, t)-\alpha_{2}^{(v)} \frac{\partial u}{\partial t}(v, t-\tau) & t>0, \forall v \in \mathcal{V}_{c} \\ \sum_{j \in \mathcal{E}_{v}} \frac{\partial u_{j}}{\partial n_{j}}(v, t)=0 & t>0, \forall v \in \mathcal{V}_{\mathrm{int}} \backslash \mathcal{V}_{\mathrm{int}}^{c} \\ u_{j_{v}}(v, t)=0 & t>0, \forall v \in \mathcal{D} \\ \frac{\partial u_{j_{v}}}{\partial n_{j_{v}}}(v, t)=0 & t>0, \forall v \in \mathcal{N} \\ u(t=0)=u^{(0)}, \frac{\partial u}{\partial t}(t=0)=u^{(1)} & \forall v \in \mathcal{V}_{c}, 0<t<\tau, \\ \frac{\partial u}{\partial t}(v, t-\tau)=f_{v}^{0}(t-\tau) & \end{cases}
$$

where $\alpha_{i}^{(v)} \geq 0$ are fixed non-negative real numbers and the delay $\tau$ is positive. 
To rewrite this system in the form (1.2), we introduce

$$
H=L^{2}(\mathcal{R})=\left\{u: \mathcal{R} \rightarrow \mathbb{R} ; u_{j} \in L^{2}\left(0, l_{j}\right), \forall j=1, \ldots, N\right\}
$$

and the operator

$$
A:\left\{\begin{array}{ccc}
D(A) & \rightarrow & H \\
\left(\varphi_{j}\right)_{j} & \mapsto & \left(-\frac{\mathrm{d}^{2}}{\mathrm{~d} x^{2}} \varphi_{j}\right)_{j}
\end{array}\right.
$$

where

$$
D(A)=\left\{\varphi \in V \cap \prod_{j=1}^{N} H^{2}\left(0, l_{j}\right) ; \sum_{j \in \mathcal{E}_{v}} \frac{\partial \varphi_{j}}{\partial n_{j}}(v)=0, \forall v \in \mathcal{V}_{\mathrm{int}} ; \frac{\partial \varphi_{j_{v}}}{\partial n_{j_{v}}}(v)=0, \forall v \in \mathcal{N} \cup \mathcal{V}_{\mathrm{ext}}^{c}\right\}
$$

and

$$
V:=\left\{\varphi \in \prod_{j=1}^{N} H^{1}\left(0, l_{j}\right): \varphi_{j}(v)=\varphi_{k}(v) \forall j, k \in \mathcal{E}_{v}, \forall v \in \mathcal{V}_{\mathrm{int}} ; \varphi_{j_{v}}(v)=0, \forall v \in \mathcal{D}\right\} .
$$

The operator $A$ is self-adjoint and positive with a compact inverse in $H$. Moreover

$$
D\left(A^{\frac{1}{2}}\right)=V .
$$

We now define $U=U_{1}=U_{2}=\mathbb{R}^{V_{c}}$, where $V_{c}$ is the cardinal of $\mathcal{V}_{c}$, with norm $\|\cdot\|_{U}=\|\cdot\|_{2}$ and the operators $B_{i}$ for $i=1,2$ as

$$
B_{i}:\left\{\begin{array}{ccc}
U & \rightarrow & D\left(A^{\frac{1}{2}}\right)^{\prime} \\
\left(k_{v}\right)_{v \in \mathcal{V}_{c}} & \mapsto \sum_{v \in \mathcal{V}_{c}} \sqrt{\alpha_{i}^{(v)}} k_{v} \delta_{v}
\end{array}\right.
$$

It is easy to verify that $B_{i}^{*}(\varphi)=\left(\sqrt{\alpha_{i}^{(v)}} \varphi(v)\right)_{v \in \mathcal{V}_{c}}^{T}$ for $\varphi \in D\left(A^{\frac{1}{2}}\right)$ and thus $B_{i} B_{i}^{*}(\varphi)=\sum_{v \in \mathcal{V}_{c}} \alpha_{i}^{(v)} \varphi(v) \delta_{v}$ for $\varphi \in D\left(A^{\frac{1}{2}}\right)$. Hence the system (7.1) can be rewritten in the form (1.2).

We notice that $(2.5)$ is here reduced to

$$
\exists 0<\alpha \leq 1, \forall \varphi \in V, \sum_{v \in \mathcal{V}_{c}}\left(\alpha_{2}^{(v)} \varphi(v)\right)^{2} \leq \alpha \sum_{v \in \mathcal{V}_{c}}\left(\alpha_{1}^{(v)} \varphi(v)\right)^{2},
$$

and therefore, the system (7.1) is well posed for $\alpha_{2}^{(v)} \leq \alpha_{1}^{(v)}$ for all $v \in \mathcal{V}_{c}$ by Theorem 2.1, and the energy is decreasing for $\alpha_{2}^{(v)}<\alpha_{1}^{(v)}$ for all $v \in \mathcal{V}_{c}$ by Proposition 3.1.

By Proposition 6.2 of [7], the generalized gap condition (6.1) holds with $M=N+1$.

We know, by [17], that the hypothesis (4.1) is satisfied. Moreover, the hypothesis (5.2) is verified because

$$
\begin{aligned}
\left\|\omega_{0}\right\|_{V}^{m+1} & \leq C\left\|\omega_{0}\right\|_{X}^{m}\left\|\omega_{0}\right\|_{D\left(A^{\frac{1-m}{2}}\right)} \\
& \leq C\left\|\left(\omega_{0}, \omega_{1}, z\right)\right\|_{D(\mathcal{A})}^{m}\left\|\omega_{0}\right\|_{D\left(A^{\frac{1-m}{2}}\right)}
\end{aligned}
$$

where $X=V \cap\left(\prod_{j=1}^{N} H^{2}\left(0, l_{j}\right)\right)$, by using Corollary 6.4 of $[17]$.

Now we define $\Psi_{k_{n}}(v)$ the matrix of size $k \times L_{n}$ by: for all $i=1, \ldots, k$, we set

$$
\left(\Psi_{k_{n}}(v)\right)_{i j}= \begin{cases}\varphi_{k_{n+i-1}+j-L_{n, i-1}}(v) & \text { if } L_{n, i-1}<j<L_{n, i}, \\ 0 & \text { else }\end{cases}
$$


where $L_{n, 0}=1$ and $L_{n, i}=\sum_{i^{\prime}=1}^{i}\left(l_{n+i^{\prime}-1}-1\right)$ for $i \geq 1$. Then, for all $\xi \in \mathbb{R}^{L_{n}}$, we have

$$
\begin{aligned}
\left\|B_{k_{n}}^{-1} \Phi_{k_{n}} \xi\right\|_{U_{1}, 2}^{2} & =\sum_{v \in \mathcal{V}_{c}} \alpha_{1}^{l}\left\|B_{k_{n}}^{-1} \Psi_{k_{n}}(v) \xi\right\|_{2}^{2} \\
& =\sum_{v \in \mathcal{V}_{c}} \alpha_{1}^{l} \xi^{T} \Psi_{k_{n}}(v)^{T} B_{k_{n}}^{-T} B_{k_{n}}^{-1} \Psi_{k_{n}}(v) \xi \\
& =\xi^{T}\left(\sum_{v \in \mathcal{V}_{c}} \alpha_{1}^{l} \Psi_{k_{n}}(v)^{T} B_{k_{n}}^{-T} B_{k_{n}}^{-1} \Psi_{k_{n}}(v)\right) \xi
\end{aligned}
$$

Therefore by setting

$$
\tilde{\mathcal{M}}^{k_{n}}=\sum_{v \in \mathcal{V}_{c}} \alpha_{1}^{l} \Psi_{k_{n}}(v)^{T} B_{k_{n}}^{-T} B_{k_{n}}^{-1} \Psi_{k_{n}}(v)
$$

we see that assumption (6.7) becomes

$$
\exists \gamma>0, \forall k \in\{1, \ldots, M\}, \forall k_{n} \in A_{k}, \lambda_{\min }\left(\tilde{\mathcal{M}}^{k_{n}}\right) \geq \gamma
$$

and the assumption (6.10) becomes

$$
\exists m \in \mathbb{R}_{+}^{*}, \exists \gamma>0, \forall k \in\{1, \ldots, M\}, \forall k_{n} \in A_{k}, \lambda_{\min }\left(\tilde{\mathcal{M}}^{k_{n}}\right) \geq \frac{\gamma}{\lambda_{k_{n}}^{2 m}}
$$

which corresponds respectively to the conditions (6.8) and (7.4) from the paper [17], because $\lambda_{k} \sim \frac{k \pi}{L}$, where $L=\sum_{j=1}^{N} l_{j}$.

Note that if the standard gap condition (6.4) holds and if all eigenvalues are simple (i.e., $l_{k}=1$ ), then the condition (6.7) becomes

$$
\exists \gamma>0, \forall k \geq 1, \sum_{v \in \mathcal{V}_{c}} \alpha_{1}^{(v)}\left|\varphi_{k}(v)\right|^{2} \geq \gamma,
$$

while the conditions (6.10) becomes

$$
\exists m \in \mathbb{R}_{+}^{*}, \exists \gamma>0, \forall k \geq 1, \sum_{v \in \mathcal{V}_{c}} \alpha_{1}^{(v)}\left|\varphi_{k}(v)\right|^{2} \geq \frac{\gamma}{\lambda_{k}^{2 m}}
$$

Consequently, we find again all the results from [17] (see for instance the examples treated in Sect. 7 of [17]), here we can even precise the dependence of the decay rate with respect to the delay $\tau$.

\subsection{An Euler-Bernoulli beam with interior damping}

We consider an Euler-Bernoulli beam of length 1 with interior damping and a delay term at $\xi$. Two types of boundary conditions will be considered. Without delay, these two problems were analyzed in $[2,3]$, where some decay rates similar to the ones proved below were obtained. 


\subsubsection{Mixed boundary conditions}

We consider the following initial and boundary system:

$$
\left\{\begin{array}{cc}
\frac{\partial^{2} \omega}{\partial t^{2}}(x, t)+\frac{\partial^{4} \omega}{\partial x^{4}}(x, t)+\alpha_{1} \frac{\partial \omega}{\partial t}(\xi, t) \delta_{\xi}+\alpha_{2} \frac{\partial \omega}{\partial t}(\xi, t-\tau) \delta_{\xi}=0 & 0<x<1, t>0 \\
\omega(0, t)=\frac{\partial \omega}{\partial x}(1, t)=\frac{\partial^{2} \omega}{\partial x^{2}}(0, t)=\frac{\partial^{3} \omega}{\partial x^{3}}(1, t)=0 & t>0 \\
\omega(x, 0)=\omega_{0}(x), \frac{\partial \omega}{\partial t}(x, 0)=\omega_{1}(x) & 0<x<1 \\
\frac{\partial \omega}{\partial t}(\xi, t-\tau)=f^{0}(t-\tau) & 0<t<\tau
\end{array}\right.
$$

where $\xi \in(0,1), \alpha_{1}, \alpha_{2}>0$ and $\tau>0$. To enter into the framework of Section 1 , we rewrite this system in the form (1.2). For that purpose, we introduce $H=L^{2}(0,1)$ and the operator

$$
A: D(A) \rightarrow H: \varphi \mapsto \frac{\mathrm{d}^{4}}{\mathrm{~d} x^{4}} \varphi
$$

where $D(A)=\left\{\varphi \in H^{4}(0,1) ; \varphi(0)=\frac{\partial \varphi}{\partial x}(1)=\frac{\partial^{2} \varphi}{\partial x^{2}}(0)=\frac{\partial^{3} \varphi}{\partial x^{3}}(1)=0\right\}$. The operator $A$ is self-adjoint and positive with a compact inverse in $H$. We now define $U=U_{1}=U_{2}=\mathbb{R}$ and the operators $B_{1}$ and $B_{2}$ as

$$
B_{i}: U \rightarrow D\left(A^{\frac{1}{2}}\right)^{\prime}: k \mapsto \sqrt{\alpha_{i}} k \delta_{\xi}, i=1,2
$$

It is easy to verify that $B_{i}^{*}(\varphi)=\sqrt{\alpha_{i}} \varphi(\xi)$ for $\varphi \in D\left(A^{\frac{1}{2}}\right)$ and thus $B_{i} B_{i}^{*}(\varphi)=\alpha_{i} \varphi(\xi) \delta_{\xi}$ for $\varphi \in D\left(A^{\frac{1}{2}}\right)$ and $i=1,2$. Then the system (7.7) can be rewritten in the form (1.2). We notice that (2.5) is equivalent to

$$
\exists 0<\alpha \leq 1, \alpha_{2} \leq \alpha \alpha_{1}
$$

and consequently, this system is well posed for $\alpha_{2} \leq \alpha_{1}$ by Theorem 2.1, and the energy is decreasing for $\alpha_{2}<\alpha_{1}$ by Proposition 3.1.

Let us now state the next well-known results about the spectral properties of $A$.

Proposition 7.1. The eigenvalues of the operator A defined in (7.8) are simple and are given by $\lambda_{k}^{2}=\left(\frac{2 k+1}{2} \pi\right)^{4}$ of associated eigenvector $\varphi_{k}(x)=\sqrt{2} \sin \left(\frac{2 k+1}{2} \pi x\right)$, for all $k \in \mathbb{N}$. Consequently the standard gap condition (6.4) holds, i.e., there exists a constant $\gamma_{0}>0$ such that

$$
\lambda_{k+1}-\lambda_{k} \geq \gamma_{0}>0 \quad \forall k \geq 0
$$

and moreover for all $k \geq 0,\left\|B_{1}^{*} \varphi_{k}\right\|_{U_{1}}=\sqrt{2 \alpha_{1}}\left|\sin \left(\left(k \pi+\frac{\pi}{2}\right) \xi\right)\right|$.

The hypothesis (4.1) was verified in [2]. Moreover, we have by Lemma 2.9 of [19]:

Lemma 7.2. $\xi$ is a rational number with an irreductible fraction

$$
\xi=\frac{p}{q}, \text { where } p \text { is odd }
$$

if and only if there exists a constant $\gamma>0$ such that

$$
\forall k \geq 1,\left|\sin \left(\left(k \pi+\frac{\pi}{2}\right) \xi\right)\right|>\gamma
$$


Therefore, by applying Proposition 3.4 and Theorem 4.4, we obtain the following results:

Proposition 7.3. Assume that $\alpha_{2}<\alpha_{1}$. Then

(i) The energy of system (7.7) decays to 0 if and only if

$$
\xi \neq \frac{2 m}{2 k+1}, m, k \in \mathbb{N} .
$$

(ii) The energy of system (7.7) decays exponentially if $\xi$ is a rational number with an irreductible fraction

$$
\xi=\frac{p}{q}, \text { where } p \text { is odd. }
$$

Remark 7.4. As mentioned before, in the case $\alpha_{2}=0$ we recover the results from [2].

\subsubsection{Other boundary conditions}

We here consider the following initial and boundary system:

$$
\left\{\begin{array}{cc}
\frac{\partial^{2} \omega}{\partial t^{2}}(x, t)+\frac{\partial^{4} \omega}{\partial x^{4}}(x, t)+\alpha_{1} \frac{\partial \omega}{\partial t}(\xi, t) \delta_{\xi}+\alpha_{2} \frac{\partial \omega}{\partial t}(\xi, t-\tau) \delta_{\xi}=0 & 0<x<1, t>0 \\
\omega(0, t)=\omega(1, t)=\frac{\partial^{\omega} \omega}{\partial x^{2}}(0, t)=\frac{\partial^{2} \omega}{\partial x^{2}}(1, t)=0 & t>0 \\
\omega(x, 0)=\omega^{0}(x), \frac{\partial \omega}{\partial t}(x, 0)=\omega^{1}(x) & 0<x<1 \\
\frac{\partial \omega}{\partial t}(\xi, t-\tau)=f^{0}(t-\tau) & 0<t<\tau,
\end{array}\right.
$$

where $\xi \in(0,1), \alpha_{1}, \alpha_{2}>0$ and $\tau>0$. This system (7.10) is not exponentially stable if $\alpha_{2}=0$ as shown in [3]. Hence we only consider the polynomial decay of system (7.10). As before we rewrite this system in the form (1.2) by introducing $H=L^{2}(0,1)$ and the operator

$$
A: D(A) \rightarrow H: \varphi \mapsto \frac{\mathrm{d}^{4}}{\mathrm{~d} x^{4}} \varphi
$$

with $D(A)=\left\{\varphi \in H^{4}(0,1) \cap V ; \frac{\partial^{2} \varphi}{\partial x^{2}}(0)=\frac{\partial^{2} \varphi}{\partial x^{2}}(1)=0\right\}, V=H^{2}(0,1) \cap H_{0}^{1}(0,1)$. The operator $A$ is self-adjoint and positive with a compact inverse in $H$. We then define $U=U_{1}=U_{2}=\mathbb{R}$ and the operators $B_{1}, B_{2}$ by (7.9).

Then the system (7.10) can be rewritten in the form (1.2) and consequently, this system is well posed for $\alpha_{2} \leq \alpha_{1}$ by Theorem 2.1, and the energy is decreasing for $\alpha_{2}<\alpha_{1}$ by Proposition 3.1.

The spectral properties of $A$ are well-known and can be summarized as follows:

Proposition 7.5. The eigenvalues of the operator $A$ defined in (7.11) are simple and given by $\lambda_{k}^{2}=k^{4} \pi^{4}$ of eigenvector $\varphi_{k}(x)=\sqrt{2} \sin (k \pi x)$, for all $k \in \mathbb{N}^{*}$. Therefore there exists a constant $\gamma_{0}>0$ such that the standard gap condition (6.4) is verified and moreover

$$
\left\|B_{1}^{*} \varphi_{k}\right\|_{U_{1}}=\sqrt{2 \alpha_{1}}|\sin (k \pi \xi)| .
$$

Hypothesis (4.1) was verified in [3]. Let us prove that the condition (5.2) is satisfied.

Lemma 7.6. Let $m \in \mathbb{R}_{+}^{*}$. Then there exists $C>0$ such that for all $\omega_{0} \in X=\left\{u \in V:\left.u\right|_{(0, \xi)} \in H^{4}(0, \xi)\right.$, $\left.\left.u\right|_{(\xi, 1)} \in H^{4}(\xi, 1), \frac{\partial^{2} u}{\partial x^{2}}(0)=\frac{\partial^{2} u}{\partial x^{2}}(1)=0\right\}$, we have

$$
\left\|\omega_{0}\right\|_{V}^{m+1} \leq C\left\|\omega_{0}\right\|_{X}^{m}\left\|\omega_{0}\right\|_{D\left(A^{\frac{1-m}{2}}\right)}
$$

where the natural norm in $X$ is given by $\|u\|_{X}^{2}=\|u\|_{H^{4}(0, \xi)}^{2}+\|u\|_{H^{4}(\xi, 1)}^{2}$. 
Proof. Let us fix a cut-off function $\eta \in \mathcal{D}(0,1)$ such that $\eta=1$ in a neighbourhood of $\xi, \eta=0$ on $\left[\frac{2}{3}+\frac{\xi}{3}, 1\right]$ and $\eta=0$ on $\left[0, \frac{\xi}{3}\right]$. Since $(1-\eta) \omega_{0} \in D(A)$, by Lemma 5.2 , we have

$$
\left\|(1-\eta) \omega_{0}\right\|_{V}^{m+1} \leq C\left\|(1-\eta) \omega_{0}\right\|_{X}^{m}\left\|(1-\eta) \omega_{0}\right\|_{D\left(A^{\frac{1-m}{2}}\right)} .
$$

Since

for some $C>0$ (depending on $\eta$ ) we get

$$
\begin{aligned}
& \left\|(1-\eta) \omega_{0}\right\|_{D\left(A^{\frac{1-m}{2}}\right)}=\sup _{\varphi \in D\left(A^{\frac{m-1}{2}}\right)} \frac{\left((1-\eta) \omega_{0}, \varphi\right)}{\left.\|\varphi\|_{D(A}^{\frac{m-1}{2}}\right)} \\
& =\sup _{\varphi \in D\left(A^{\frac{m-1}{2}}\right)} \frac{\left(\omega_{0},(1-\eta) \varphi\right)}{\left.\|\varphi\|_{D(A} \frac{m-1}{2}\right)} \\
& \leq C\left\|\omega_{0}\right\|_{D\left(A^{\frac{1-m}{2}}\right)} \text {, }
\end{aligned}
$$

$$
\left\|(1-\eta) \omega_{0}\right\|_{V}^{m+1} \leq C\left\|\omega_{0}\right\|_{X}^{m}\left\|\omega_{0}\right\|_{D\left(A^{\frac{1-m}{2}}\right)} .
$$

In a second step, we set

$$
\begin{array}{ll}
\omega_{1}(x)=\eta(\xi-x) \omega_{0}(\xi-x) & \text { if } 0<x<l_{1}:=\xi \\
\omega_{2}(x)=\eta(x+\xi) \omega_{0}(x+\xi) & \text { if } 0<x<l_{2}:=1-\xi .
\end{array}
$$

For any $j=1,2$, we introduce the following extension of $\omega_{j}$ :

$$
\begin{array}{cl}
(E \omega)_{j}(x)=\omega_{j}(x) & \text { if } x \in\left(0, l_{j}\right), \\
(E \omega)_{-j}(x)=\sum_{i=0}^{n-1} \nu_{i} \omega_{j}\left(-2^{i} x\right) & \text { if } x \in\left(-2^{-(n-1)} l_{j}, 0\right),
\end{array}
$$

where $\omega_{j}$ is extended by zero outside its support and the real numbers $\nu_{i}$ are the unique solution of the system

$$
\left\{\begin{array}{l}
\sum_{i=0}^{n-1} \nu_{i}=1 \\
-\sum_{i=0}^{n-1} 2^{i} \nu_{i}=1 \\
\sum_{i=0}^{n-1} 2^{2 i} \nu_{i}=1 \\
-\sum_{i=0}^{n-1} 2^{3 i} \nu_{i}=1 \\
\sum_{i=0}^{n-1} 2^{-2 k i} \nu_{i}=1, \forall k=1, \ldots, n-4
\end{array}\right.
$$

and finally $n \in \mathbb{N}^{*}$ is chosen large enough such that $n \geq m+3$.

We obtain an extension of $\omega$ to a function $E \omega$, which belongs to $\mathcal{D}(\tilde{A})$ (due to the four first properties of the $\nu_{i}$ ), where $\tilde{A}$ is the positive operator $\frac{\mathrm{d}^{4}}{\mathrm{~d} x^{4}}$ on the star shaped network $\tilde{\mathcal{S}}=\cup_{j=1,2}\left(0, l_{j}\right) \cup \cup_{j=1,2}\left(-2^{-(n-1)} l_{j}, 0\right)$, with interior vertex $\xi$ (identified to 0 ) and Dirichlet boundary conditions at all other vertices.

Therefore, we can apply the interpolation lemma 5.2 to $\tilde{A}$ and then write

$$
\|E \omega\|_{D\left(\tilde{A}^{\frac{1}{2}}\right)}^{m+1} \leq C\|E \omega\|_{D(\tilde{A})}^{m}\|E \omega\|_{D\left(\tilde{A}^{\frac{1-m}{2}}\right)} .
$$

But we easily check that

Consequently, we have

$$
\left\|\eta \omega_{0}\right\|_{D\left(A^{\frac{1}{2}}\right)}^{m+1} \leq\|E \omega\|_{D\left(\tilde{A}^{\frac{1}{2}}\right)}^{m+1}
$$

$$
\left\|\eta \omega_{0}\right\|_{D\left(A^{\frac{1}{2}}\right)}^{m+1} \leq C\|E \omega\|_{D(\tilde{A})}^{m}\|E \omega\|_{D\left(\tilde{A}^{\frac{1-m}{2}}\right)} .
$$


Moreover, as $E$ is an extension operator, we have

$$
\|E \omega\|_{D(\tilde{A})} \leq K\left\|\omega_{0}\right\|_{X}
$$

and thus

$$
\left\|\eta \omega_{0}\right\|_{D\left(A^{\frac{1}{2}}\right)}^{m+1} \leq C\left\|\omega_{0}\right\|_{X}^{m}\|E \omega\|_{D\left(\tilde{A}^{\frac{1-m}{2}}\right)} .
$$

To estimate the last factor, we use a duality argument. We write

$$
\|E \omega\|_{D\left(\tilde{A}^{\frac{1-m}{2}}\right)}=\sup _{\varphi \in D\left(\tilde{A}^{\frac{m-1}{2}}\right)} \frac{|(E \omega, \varphi)|}{\|\varphi\|_{D\left(\tilde{A}^{\frac{m-1}{2}}\right)}} .
$$

For $\varphi \in D\left(\tilde{A}^{\frac{m-1}{2}}\right)$, we have

$$
\int_{\tilde{\mathcal{S}}} E \omega \varphi=\sum_{j=1,2} \int_{0}^{l_{j}} \omega_{j}(x) \varphi_{j}(x) \mathrm{d} x+\sum_{j=1,2} \int_{-2^{-(n-1)} l_{j}}^{0}(E \omega)_{-j}(x) \varphi_{-j}(x) \mathrm{d} x .
$$

By changes of variables, we obtain

$$
\int_{\tilde{\mathcal{S}}} E \omega \varphi=\sum_{j=1,2} \int_{0}^{l_{j}} \omega_{j}(x)(F \varphi)_{j}(x) \mathrm{d} x,
$$

where

$$
(F \varphi)_{j}(x)=\varphi_{j}(x)+\chi_{j}(x) \sum_{i=0}^{n-1} \nu_{i} 2^{-i} \varphi_{j}\left(-2^{-i} x\right) \quad \forall x \in\left(0, l_{j}\right),
$$

the cut-off function $\chi_{j}$ being fixed such that $\chi_{j} \equiv 1$ on $\left[0,2 l_{j} / 3\right]$ and $\chi_{j} \equiv 0$ on $\left[5 l_{j} / 6, l_{j}\right]$ (reminding that $\omega_{j}(x) \equiv 0$ for $\left.x>2 l_{j} / 3\right)$. Now we notice that the conditions on $\nu_{i}$ guarantees that $F \varphi$ belongs to $D\left(A^{\frac{m-1}{2}}\right)$ and by Leibniz's rule we have

Therefore

$$
\|F \varphi\|_{D\left(A \frac{m-1}{2}\right)} \leq C\|\varphi\|_{D\left(\tilde{A}^{\frac{m-1}{2}}\right)} .
$$

By duality, we conclude that

$$
\int_{\tilde{\mathcal{S}}} E \omega \varphi \leq C\|\omega\|_{D\left(A^{\frac{1-m}{2}}\right)}\|\varphi\|_{D\left(\tilde{A}^{\frac{m-1}{2}}\right)} .
$$

Consequently, with the previous inequalities, we obtain

$$
\|E \omega\|_{D\left(\tilde{A}^{\frac{1-m}{2}}\right)} \leq C\|\omega\|_{D\left(A^{\frac{1-m}{2}}\right)} .
$$

$$
\left\|\eta \omega_{0}\right\|_{D\left(A^{\frac{1}{2}}\right)}^{m+1} \leq C\|\omega\|_{X}^{m}\|\omega\|_{D\left(A^{\frac{1-m}{2}}\right)} .
$$

The conclusion follows from (7.12) and (7.13).

This lemma leads to (5.2). Indeed for $\left(\omega_{0}, \omega_{1}, z\right) \in D(\mathcal{A})$, we have

$$
\begin{aligned}
\left\|\omega_{0}\right\|_{V}^{m+1} & \left.\leq C\left\|\omega_{0}\right\|_{X}^{m}\left\|\omega_{0}\right\|_{D(A} \frac{1-m}{2}\right) \\
& \leq C\left\|\left(\omega_{0}, \omega_{1}, z\right)\right\|_{D(\mathcal{A})}^{m}\left\|\omega_{0}\right\|_{D\left(A^{\frac{1-m}{2}}\right.}
\end{aligned}
$$

Now, we denote by $\mathcal{S}$ the set of all real numbers $\rho$ such that $\rho \notin \mathbb{Q}$ and if $\left[0, a_{1}, \ldots, a_{n}, \ldots\right]$ is the expansion of $\rho$ as a continued fraction, then the sequence $\left(a_{n}\right)$ is bounded. It is well-known that $\mathcal{S}$ is uncountable and that its Lebesgue measure is zero. Roughly speaking, the set $\mathcal{S}$ contains all irrational numbers which are badly 
approximated by rational numbers. In particular, by the Euler-Lagrange theorem, $\mathcal{S}$ contains all irrational quadratic numbers (i.e. the roots of a second order equation with rational coefficients). By a classical result, we have:

Lemma 7.7. If $s \in \mathcal{S}$, then there exists a positive constant $\gamma$ such that

$$
|\sin (k \pi s)| \geq \frac{\gamma}{k} \quad \forall k \geq 1 .
$$

Therefore, by applying Proposition 3.4 and Theorem 5.3 with $m=\frac{1}{2}$, we obtain the next results:

Proposition 7.8. Assume that $\alpha_{2}<\alpha_{1}$. Then

(i) The energy of system (7.10) decays to 0 if and only if $\xi$ is irrational.

(ii) The energy of system (7.10) decays polynomially like $\frac{1}{(1+t)^{2}}$ if $\xi$ belongs to $\mathcal{S}$.

Remark 7.9. Again in the case $\alpha_{2}=0$ we recover the results from $[2,3]$.

\subsection{Examples with distributed damping terms}

7.3.1. A non homogeneous string with distributed damping terms (1-d)

We consider the following initial and boundary system:

$$
\left\{\begin{array}{cl}
\frac{\partial^{2} \omega}{\partial t^{2}}(x, t)-\frac{\partial^{2} \omega}{\partial x^{2}}(x, t)+\alpha_{1} \frac{\partial \omega}{\partial t}(x, t) \chi_{\mid I_{1}}+\alpha_{2} \frac{\partial \omega}{\partial t}(x, t-\tau) \chi_{\mid I_{2}}=0 & \text { in }(0,1) \times(0, \infty) \\
\omega(0, t)=\omega(1, t)=0 & t>0 \\
\omega(x, 0)=\omega_{0}(x), \frac{\partial \omega}{\partial t}(x, 0)=\omega_{1}(x) & \text { in }(0,1) \\
\frac{\partial \omega}{\partial t}(x, t-\tau)=f^{0}(x, t-\tau) & \text { in } I_{2} \times(0, \tau),
\end{array}\right.
$$

where here and below $\chi_{\mid I}$ denotes the characteristic function of the set $I$. In the remainder of this subsection we assume that $\alpha_{1}, \alpha_{2}>0, \tau>0$ and

$$
I_{2} \subset I_{1} \subset[0,1]
$$

Later we will need that

$$
\exists \delta \in[0,1] \text { and } \epsilon>0:[\delta, \delta+\epsilon] \subset I_{1} .
$$

We rewrite this system in the form (1.2). For that purpose, we introduce $H=L^{2}(0,1)$ and the operator

$$
A: D(A) \rightarrow H: \varphi \mapsto-\frac{\mathrm{d}^{2}}{\mathrm{~d} x^{2}} \varphi
$$

where $D(A)=H_{0}^{1}(0,1) \cap H^{2}(0,1)$ and $V=D\left(A^{\frac{1}{2}}\right)=H_{0}^{1}(0,1)$. The operator $A$ is self-adjoint and positive with a compact inverse in $H$. We then define $U_{i}=L^{2}\left(I_{i}\right)$ and the operators $B_{i}$ as

$$
B_{i}: U_{i} \rightarrow H \subset V^{\prime}: k \mapsto \sqrt{\alpha_{i}} \tilde{k} \chi_{\mid I_{i}}
$$

where $\tilde{k}$ is the extension of $k$ by zero outside $I_{i}$ (which defines an element of $L^{2}(0,1)$ ).

It is easy to verify that $B_{i}^{*}(\varphi)=\sqrt{\alpha_{i}} \varphi_{\mid I_{i}}$ for $\varphi \in V$ and thus $B_{i} B_{i}^{*}(\varphi)=\alpha_{i} \varphi_{\mid I_{i}} \chi_{\mid I_{i}}=\alpha_{i} \varphi \chi_{\mid I_{i}}$ for $\varphi \in V$ and $i=1,2$. Then the system (7.14) can be rewritten in the form (1.2). Moreover

$$
\left\|B_{i}^{*} \varphi\right\|_{U_{i}}^{2}=\alpha_{i} \int_{I_{i}}|\varphi|^{2} \mathrm{~d} x .
$$


Therefore, we notice that (2.5) is equivalent to

$$
\exists 0<\alpha \leq 1, \alpha_{2} \int_{I_{2}}|\varphi|^{2} \mathrm{~d} x \leq \alpha \alpha_{1} \int_{I_{1}}|\varphi|^{2} \mathrm{~d} x
$$

and consequently, this system is well posed for $\alpha_{2} \leq \alpha_{1}$ by Theorem 2.1, and the energy is decreasing for $\alpha_{2}<\alpha_{1}$ by Proposition 3.1.

Proposition 7.10. The eigenvalues of the operator $A$ defined in (7.16) are simple and given by $\lambda_{k}^{2}=(k \pi)^{2}$ of associated eigenvector $\varphi_{k}(x)=\sqrt{2} \sin (k \pi x)$, for all $k \in \mathbb{N}^{*}$. Hence there exists a constant $\gamma_{0}>0$ such that the standard gap condition (6.4) holds. Moreover if (7.15) holds, then there exists $\gamma>0$ such that $\forall k \geq 1,\left\|B_{1}^{*} \varphi_{k}\right\|_{U_{1}} \geq \gamma$.

Proof. It is well-known that the eigenvectors of the operator $A$ are $\varphi_{k}(x)=\sqrt{2} \sin (k \pi x)$ of eigenvalue $(k \pi)^{2}, k \geq 1$ of multiplicity 1 . Hence, the standard gap condition (6.4) is verified.

From the definition of $B_{1}^{*}$ we have

$$
\begin{aligned}
\left\|B_{1}^{*} \varphi_{k}\right\|_{U_{1}}^{2} & =\alpha_{1} \int_{I_{1}}\left|\varphi_{k}(x)\right|^{2} \mathrm{~d} x \\
& \geq 2 \alpha_{1} \int_{\delta}^{\delta+\epsilon}|\sin (k \pi x)|^{2} \mathrm{~d} x=\alpha_{1}\left[x-\frac{\sin (2 k \pi x)}{2 k \pi}\right]_{\delta}^{\delta+\epsilon} \\
& \geq \alpha_{1}\left(\epsilon-\frac{1}{k \pi}\right) \geq \alpha_{1} \frac{\epsilon}{2},
\end{aligned}
$$

for $k \geq \mathbf{E}\left(\frac{2}{\epsilon \pi}\right)+1=: k_{\epsilon}$, where $\mathbf{E}(x)$ is the entire part of $x$. This leads to the conclusion because for $k \in\left\{1, \ldots, k_{\epsilon}\right\}$, we have

$$
\int_{\delta}^{\delta+\epsilon}|\sin (k \pi x)|^{2} \mathrm{~d} x>0
$$

Lemma 7.11. The operators $A$ and $B=\left(B_{1} B_{2}\right) \in \mathcal{L}\left(U, V^{\prime}\right)$ where $U=U_{1} \times U_{2}$ satisfy assumption (4.1).

Proof. Let $i \in\{1,2\}$ and $\varphi \in L^{2}\left(I_{i}\right)$. It can be easily checked that $v=\left(\lambda^{2}+A\right)^{-1} B_{i} \varphi$ satisfies

$$
\left\{\begin{array}{c}
\lambda^{2} v-\frac{\mathrm{d}^{2} v}{\mathrm{~d} x^{2}}=\sqrt{\alpha_{i}} \tilde{\varphi} \chi_{\mid I_{i}} \\
v(0)=v(1)=0
\end{array}\right.
$$

As $v \in L^{2}(0,1), v$ can be written as

$$
v=\sum_{k=1}^{\infty} c_{k} \varphi_{k}
$$

By replacing $v$ in (7.18), $c_{k}$ must satisfy

$$
c_{k}=\frac{\sqrt{\alpha_{i}}}{\lambda^{2}+\lambda_{k}^{2}}\left(\tilde{\varphi}, \varphi_{k}\right)
$$

and therefore

$$
v=\sum_{k=1}^{\infty} \frac{\sqrt{\alpha_{i}}}{\lambda^{2}+\lambda_{k}^{2}}\left(\tilde{\varphi}, \varphi_{k}\right) \varphi_{k} .
$$

Moreover

$$
\|\lambda v\|_{L^{2}(0,1)}^{2}=\sum_{k=1}^{\infty}\left|\frac{\lambda}{\lambda^{2}+\lambda_{k}^{2}}\right|^{2} \alpha_{i}\left|\left(\tilde{\varphi}, \varphi_{k}\right)\right|^{2} .
$$

Now, we set $z=\frac{\lambda}{\lambda^{2}+\lambda_{k}^{2}}$ and, if $\lambda=\beta+\mathrm{i} y$, with $y \in \mathbb{R}$, then

$$
|z|^{2}=\frac{\beta^{2}+y^{2}}{\left(\beta^{2}-y^{2}+\lambda_{k}^{2}\right)^{2}+4 \beta^{2} y^{2}} \leq C(\beta),
$$


where $C(\beta)$ is a positive constant depending only on $\beta$. Indeed, if $y^{2} \leq \frac{\beta^{2}+\lambda_{k}^{2}}{2}$, then

$$
|z|^{2} \leq \frac{\beta^{2}+y^{2}}{\left(\frac{\beta^{2}+\lambda_{k}^{2}}{2}\right)^{2}} \leq \frac{\beta^{2}+\frac{\beta^{2}+\lambda_{k}^{2}}{2}}{\left(\frac{\beta^{2}+\lambda_{k}^{2}}{2}\right)^{2}}
$$

which is bounded uniformly in $k$, and if $y^{2} \geq \frac{\beta^{2}+\lambda_{k}^{2}}{2}$, then

$$
|z|^{2} \leq \frac{\beta^{2}+y^{2}}{4 \beta^{2} y^{2}}
$$

which is a decreasing function with respect to $y$ and thus

$$
|z|^{2} \leq \frac{\beta^{2}+\frac{\beta^{2}+\lambda_{k}^{2}}{2}}{4 \beta^{2}\left(\frac{\beta^{2}+\lambda_{k}^{2}}{2}\right)},
$$

which is again uniformly bounded in $k$. Therefore, we have

$$
\|\lambda v\|_{L^{2}(0,1)}^{2} \leq C(\beta) \alpha_{i} \sum_{k=1}^{\infty}\left|\left(\tilde{\varphi}, \varphi_{k}\right)\right|^{2} \leq C(\beta) \alpha_{i}\|\varphi\|_{L^{2}\left(I_{i}\right)}^{2}
$$

which leads to

$$
\left\|\lambda B_{j}^{*} v\right\|_{L^{2}\left(I_{j}\right)}^{2} \leq \alpha_{j}\|\lambda v\|_{L^{2}(0,1)}^{2} \leq C(\beta) \alpha_{i} \alpha_{j}\|\varphi\|_{L^{2}\left(I_{i}\right)}^{2}, \quad \forall j \in\{1,2\} .
$$

Consequently, the operator $\lambda \rightarrow \lambda B_{j}^{*}(\lambda I+A)^{-1} B_{i}$ is bounded on $C_{\beta}$ and the lemma is proved.

Therefore, by applying Theorem 4.4, we obtain:

Proposition 7.12. If $\alpha_{2}<\alpha_{1}$ and (7.15) holds, then the energy of system (7.14) decays exponentially.

7.3.2. The wave equation with distributed damping terms

Let $\Omega$ be an open bounded domain of $\mathbb{R}^{n}, n \geq 1$, with a boundary $\Gamma$ of class $C^{2}$. We assume that $\Gamma$ is divided into two parts $\Gamma_{D}$ and $\Gamma_{N}$, i.e. $\Gamma=\Gamma_{D} \cup \Gamma_{N}$, with $\bar{\Gamma}_{D} \cap \bar{\Gamma}_{N}=\emptyset$ and $\Gamma_{D} \neq \emptyset$. Let

$$
O_{2} \subset O_{1} \subset \Omega
$$

such that $O_{1}$ is an open neighborhood of $\Gamma_{N}\left(\right.$ i.e. $\Gamma_{N} \subset \partial O_{1}$ ). Moreover, we assume that $x_{0} \in \mathbb{R}^{n}$ is such that

$$
\left(x-x_{0}\right) \cdot \nu(x) \leq 0 \quad \forall x \in \Gamma_{D} .
$$

We consider the following initial and boundary system:

$$
\left\{\begin{array}{cl}
\frac{\partial^{2} \omega}{\partial t^{2}}(x, t)-\Delta \omega(x, t)+\alpha_{1} \frac{\partial \omega}{\partial t}(x, t) \chi_{\mid O_{1}}+\alpha_{2} \frac{\partial \omega}{\partial t}(x, t-\tau) \chi_{\mid O_{2}}=0 & \text { in } \Omega \times(0, \infty), \\
\omega(x, t)=0 & \text { on } \Gamma_{D} \times(0, \infty), \\
\frac{\partial \omega}{\partial \nu}(x, t)=0 & \text { on } \Gamma_{N} \times(0, \infty), \\
\omega(x, 0)=\omega_{0}(x), \frac{\partial \omega}{\partial t}(x, 0)=\omega_{1}(x) & \text { in } \Omega, \\
\frac{\partial \omega}{\partial t}(x, t-\tau)=f^{0}(x, t-\tau) & \text { in } O_{2} \times(0, \tau),
\end{array}\right.
$$


where $\frac{\partial \omega}{\partial \nu}$ is the normal derivative of $\omega$ and $\alpha_{1}, \alpha_{2}>0, \tau>0$. In order to reformulate this system in the form (1.2), we introduce $H=L^{2}(\Omega)$ and the operator

$$
A: D(A) \rightarrow H: \varphi \mapsto-\Delta \varphi
$$

where $D(A)=\left\{\varphi \in V \cap H^{2}(\Omega): \frac{\partial \varphi}{\partial \nu}(x)=0\right.$ on $\left.\Gamma_{N}\right\}$ and $V=D\left(A^{\frac{1}{2}}\right)=\left\{\varphi \in H^{1}(\Omega): \varphi=0\right.$ on $\left.\Gamma_{D}\right\}$. The operator $A$ is self-adjoint and positive with a compact inverse in $H$. We then define $U_{i}=L^{2}\left(O_{i}\right)$ and the operators $B_{i}$ as

$$
B_{i}: U_{i} \rightarrow H \subset V^{\prime}: k \mapsto \sqrt{\alpha_{i}} \tilde{k} \chi_{\mid O_{i}}
$$

where $\tilde{k}$ is the extension of $k$ by zero outside $O_{i}$ (which defines an element of $L^{2}(\Omega)$ ).

It is easy to verify that $B_{i}^{*}(\varphi)=\sqrt{\alpha_{i}} \varphi_{\mid O_{i}}$ for $\varphi \in V$ and thus $B_{i} B_{i}^{*}(\varphi)=\alpha_{i} \varphi_{\mid O_{i}} \chi_{\mid O_{i}}$ for $\varphi \in V$ and $i=1,2$. Then the system (7.20) can be rewritten in the form (1.2). Moreover

$$
\left\|B_{i}^{*} \varphi\right\|_{U_{i}}^{2}=\alpha_{i} \int_{O_{i}}|\varphi|^{2} \mathrm{~d} x
$$

and therefore (2.5) is equivalent to

$$
\exists 0<\alpha \leq 1, \alpha_{2} \int_{O_{2}}|\varphi|^{2} \mathrm{~d} x \leq \alpha \alpha_{1} \int_{O_{1}}|\varphi|^{2} \mathrm{~d} x .
$$

Consequently, this system is well posed for $\alpha_{2} \leq \alpha_{1}$ by Theorem 2.1, and the energy is decreasing for $\alpha_{2}<\alpha_{1}$ by Proposition 3.1.

To obtain the exponential decay of system (7.20), we simply check the observability inequality (4.6) and hypothesis (4.1), which are the aim of the two following propositions.

Proposition 7.13. There exists a time $T_{0}$ such that for all times $T>T_{0}$ there exists a positive constant $C$ (depending on $T$ ) for which the observability inequality (4.6) holds for any regular solution of system (4.3).

Proof. This proposition is proved in $[14,16]$.

Proposition 7.14. The operators $A$ and $B=\left(B_{1} B_{2}\right) \in \mathcal{L}\left(U, V^{\prime}\right)$ where $U=U_{1} \times U_{2}$ satisfy assumption (4.1).

Proof. Let $i \in\{1,2\}$ and $\varphi \in L^{2}\left(O_{i}\right)$. It can be easily checked that $v=\left(\lambda^{2}+A\right)^{-1} B_{i} \varphi$ satisfies

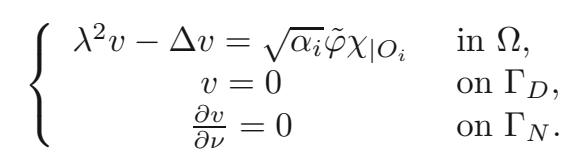

As $v \in L^{2}(\Omega)$, it can be written as

$$
v=\sum_{k=1}^{\infty} c_{k} \varphi_{k} .
$$

By replacing $v$ in (7.23), $c_{k}$ must satisfy

$$
c_{k}=\frac{\sqrt{\alpha_{i}}}{\lambda^{2}+\lambda_{k}^{2}}\left(\tilde{\varphi}, \varphi_{k}\right),
$$

and therefore

$$
v=\sum_{k=1}^{\infty} \frac{\sqrt{\alpha_{i}}}{\lambda^{2}+\lambda_{k}^{2}}\left(\tilde{\varphi}, \varphi_{k}\right) \varphi_{k} .
$$


Moreover, by Parseval's identity, we have

$$
\|\lambda v\|_{L^{2}(\Omega)}^{2}=\sum_{k=1}^{\infty}\left|\frac{\lambda}{\lambda^{2}+\lambda_{k}^{2}}\right|^{2} \alpha_{i}\left|\left(\tilde{\varphi}, \varphi_{k}\right)\right|^{2} .
$$

Now, for $\lambda=\beta+\mathrm{i} y$, with $y \in \mathbb{R}$, we have checked in the previous subsection that

$$
\left|\frac{\lambda}{\lambda^{2}+\lambda_{k}^{2}}\right|^{2} \leq C(\beta)
$$

where $C(\beta)$ is a positive constant depending only on $\beta$. Therefore, we have

$$
\|\lambda v\|_{L^{2}(\Omega)}^{2} \leq C(\beta) \alpha_{i} \sum_{k=1}^{\infty}\left|\left(\tilde{\varphi}, \varphi_{k}\right)\right|^{2} \leq C(\beta) \alpha_{i}\|\varphi\|_{L^{2}\left(O_{i}\right)}^{2},
$$

which leads to

$$
\left\|\lambda B_{j}^{*} v\right\|_{L^{2}\left(O_{j}\right)}^{2} \leq \alpha_{j}\|\lambda v\|_{L^{2}(\Omega)}^{2} \leq C(\beta) \alpha_{i} \alpha_{j}\|\varphi\|_{L^{2}\left(O_{i}\right)}^{2}, \quad \forall j \in\{1,2\} .
$$

Consequently, the operator $\lambda \rightarrow \lambda B_{j}^{*}(\lambda I+A)^{-1} B_{i}$ is bounded on $C_{\beta}$ and the lemma is proved.

Therefore, by applying Theorem 4.4, we obtain:

Proposition 7.15. If $\alpha_{2}<\alpha_{1}$, then the energy of system (7.20) decays exponentially.

Remark 7.16. This result is a generalization of [16] because in [16] the authors supposed that $O_{2}=O_{1}$.

7.3.3. A beam with distributed damping terms

We consider the following initial and boundary system:

$$
\left\{\begin{array}{cl}
\frac{\partial^{2} \omega}{\partial t^{2}}(x, t)+\frac{\partial^{4} \omega}{\partial x^{4}}(x, t)+\alpha_{1} \frac{\partial \omega}{\partial t}(x, t) \chi_{\mid I_{1}}+\alpha_{2} \frac{\partial \omega}{\partial t}(x, t-\tau) \chi_{\mid I_{2}}=0 & \text { in }(0,1) \times(0, \infty), \\
\omega(0, t)=\omega(1, t)=\frac{\partial^{2} \omega}{\partial x^{2}}(0, t)=\frac{\partial^{2} \omega}{\partial x^{2}}(1, t)=0 & t>0, \\
\omega(x, 0)=\omega_{0}(x), \frac{\partial \omega}{\partial t}(x, 0)=\omega_{1}(x) & \text { in }(0,1), \\
\frac{\partial \omega}{\partial t}(x, t-\tau)=f^{0}(x, t-\tau) & \text { in } I_{2} \times(0, \tau),
\end{array}\right.
$$

where $\alpha_{1}, \alpha_{2}>0, \tau>0$ and where

$$
I_{2} \subset I_{1} \subset[0,1]
$$

We rewrite this system in the form (1.2) by introducing $H=L^{2}(0,1)$, the operator $A$ by $(7.11)$ and the operators $B_{1}$ and $B_{2}$ by (7.17). Hence this system is well posed for $\alpha_{2} \leq \alpha_{1}$ by Theorem 2.1, and the energy is decreasing for $\alpha_{2}<\alpha_{1}$ by Proposition 3.1.

By the results of the previous subsections, we know that the standard gap condition holds and if (7.15) holds that the eigenvector $\varphi_{k}(x)=\sqrt{2} \sin (k \pi x)$ of $A$ associated with $\lambda_{k}^{2}=k^{4} \pi^{4}$ satisfies

$$
\left\|B_{1}^{*} \varphi_{k}\right\|_{U_{1}} \geq \gamma
$$

for some $\gamma>0$.

Lemma 7.17. The operators $A$ and $B=\left(B_{1} B_{2}\right) \in \mathcal{L}\left(U, V^{\prime}\right)$ where $U=U_{1} \times U_{2}$ satisfy assumption (4.1).

Proof. The proof is the same than in the previous subsection.

Therefore, by applying Proposition 3.4 and Theorem 4.4, we obtain the

Proposition 7.18. If $\alpha_{2}<\alpha_{1}$ and (7.15) holds, then the energy of system (7.24) decays exponentially. 


\subsubsection{A wave equation on 1-d networks with internal damping terms}

In this last subsection we consider again a wave equation on a given network $\mathcal{R}$ but we suppose that the feedbacks are located in the edges. Namely with the notations from Section 7.1 we consider the system:

$$
\begin{cases}\frac{\partial^{2} u_{j}}{\partial t^{2}}(x, t)-\frac{\partial^{2} u_{j}}{\partial x^{2}}(x, t)+\alpha_{1}^{(j)} \frac{\partial u_{j}}{\partial t}(x, t) \chi_{\mid I_{1}^{(j)}} & \\ +\alpha_{2}^{(j)} \frac{\partial u_{j}}{\partial t}(x, t-\tau) \chi_{\mid I_{2}^{(j)}}=0 & 0<x<l_{j}, t>0, \forall j \in\{1, \ldots, N\}, \\ u_{j}(v, t)=u_{l}(v, t)=u(v, t) & t>0, \forall v \in \mathcal{V} \backslash \mathcal{D}, \\ \sum_{j \in \mathcal{E}_{v}} \frac{\partial u_{j}}{\partial n_{j}}(v, t)=0 & t>0, \forall v \in \mathcal{D}, \\ u_{j_{v}}(v, t)=0 & \\ u(t=0)=u^{(0)}, \frac{\partial u}{\partial t}(t=0)=u^{(1)}, & \text { in } I_{2}^{(j)} \times(0, \tau), \forall j \in\{1, \ldots, N\}, \\ \frac{\partial u_{j}}{\partial t}(x, t-\tau)=f_{j}^{0}(x, t-\tau) & \text { int },\end{cases}
$$

where $\alpha_{i}^{(j)}$ are fixed non-negative real numbers, the delay $\tau$ is positive and the intervals $I_{i}^{(j)}$ satisfy

$$
I_{2}^{(j)} \subset I_{1}^{(j)} \subset e_{j}
$$

As before we can rewrite this system in the form (1.2), by introducing $H=L^{2}(\mathcal{R})$, the operator $A$ defined by $(7.2), U_{i}=L^{2}\left(\cup_{j=1}^{N} I_{i}^{(j)}\right)$ and the operators $B_{i}$ for $i=1,2$ as

$$
B_{i}:\left\{\begin{array}{rlc}
U & \rightarrow & D\left(A^{\frac{1}{2}}\right)^{\prime} \\
k & \mapsto \sum_{j=1}^{N} \sqrt{\alpha_{i}^{(j)}} \tilde{k} \chi_{\mid I_{i}^{(j)}}
\end{array}\right.
$$

where $\tilde{k}$ means the extension of $k$ by zero outside $\cup_{j=1}^{N} I_{i}^{(j)}$. It is easy to verify that

$$
B_{i}^{*}(\varphi)=\sum_{j=1}^{N} \sqrt{\alpha_{i}^{(j)}} \varphi_{\mid I_{i}^{(j)}} \chi_{\mid I_{i}^{(j)}}
$$

for $\varphi \in D\left(A^{\frac{1}{2}}\right)$. Hence the system (7.25) can be rewritten in the form (1.2).

As before it is easy to see that the system (7.1) is well posed for $\alpha_{2}^{(j)} \leq \alpha_{1}^{(j)}$ for all $j=1, \ldots, N$ by Theorem 2.1, and the energy is decreasing for $\alpha_{2}^{(j)}<\alpha_{1}^{(j)}$ for all $j=1, \ldots, N$ by Proposition 3.1.

As mentioned before by Proposition 6.2 from [7], the generalized gap condition (6.1) holds with $M=N+1$, but in this general setting, the conditions (6.7) or (6.10) seem difficult to check. Hence, for the sake of simplicity, if we suppose here that the standard gap condition (6.4) holds and that all eigenvalues are simple $\left(i . e ., l_{k}=1\right)$, then condition (6.7) becomes

$$
\exists \gamma>0, \forall k \geq 1, \sum_{j=1}^{N} \alpha_{1}^{(j)} \int_{I_{1}^{(j)}} \varphi_{k}(x)^{2} \mathrm{~d} x \geq \gamma
$$

while condition (6.10) becomes

$$
\exists m \in \mathbb{R}_{+}^{*}, \exists \gamma>0, \forall k \geq 1, \sum_{j=1}^{N} \alpha_{1}^{(j)} \int_{I_{1}^{(j)}} \varphi_{k}(x)^{2} \mathrm{~d} x \geq \frac{\gamma}{\lambda_{k}^{2 m}} .
$$


For instance using an argument like in Proposition 7.10, we easily see that (7.27) (resp. (7.28)) holds if (7.5) (resp. (7.6)) holds and if $\cup_{j=1}^{N} I_{1}^{(j)}$ contains a neighborhood of the set of control points $\mathcal{V}_{c}$.

In the same manner, we have:

Proposition 7.19. If there exists $\varepsilon>0$ and for all $j \in\{1, \ldots, N\}$, there exists $\delta_{j} \in\left(0, l_{j}\right)$ such that

$$
\left[\delta_{j}, \delta_{j}+\varepsilon\right] \subset I_{1}^{(j)} \quad \forall j \in\{1, \ldots, N\}
$$

then (7.27) holds.

Proof. For any $k \in \mathbb{N}^{*}$, let $\varphi_{k}$ be the eigenvector of $A$ associated with the eigenvalue $\lambda_{k}^{2}$. Then its restriction $\varphi_{k, j}$ to the edge $e_{j}$ can be written in the form

$$
\varphi_{k, j}(x)=c_{k, j} \cos \left(\lambda_{k} x\right)+d_{k, j} \sin \left(\lambda_{k} x\right) \quad \forall x \in\left(0, l_{j}\right),
$$

for some real numbers $c_{k, j}$ and $d_{k, j}$. Hence the normalization of $\varphi_{k}$ yields

$$
1=\sum_{j=1}^{N} \int_{0}^{l_{j}}\left|\varphi_{k, j}(x)\right|^{2} \mathrm{~d} x \leq 2\left(\max _{j} l_{j}\right) \sum_{j=1}^{N}\left(c_{k, j}^{2}+d_{k, j}^{2}\right) .
$$

On the other hand by the above expression of $\varphi_{k, j}$ and direct calculations, we see that

$$
\begin{aligned}
\int_{I_{1}^{(j)}} \varphi_{k}(x)^{2} \mathrm{~d} x & \geq \int_{\delta_{j}}^{\delta_{j}+\varepsilon} \varphi_{k}(x)^{2} \mathrm{~d} x \\
& \geq\left(c_{k, j}^{2}+d_{k, j}^{2}\right)\left(\frac{\varepsilon}{2}-\frac{1}{2 \lambda_{k}}\right)-\frac{\left|c_{k, j} d_{k, j}\right|}{\lambda_{k}} \\
& \geq\left(c_{k, j}^{2}+d_{k, j}^{2}\right)\left(\frac{\varepsilon}{2}-\frac{1}{\lambda_{k}}\right) .
\end{aligned}
$$

Therefore for $k$ large enough such that

$$
\frac{\varepsilon}{2}-\frac{1}{\lambda_{k}} \geq \frac{\varepsilon}{4}
$$

which is equivalent to $k \geq k_{\varepsilon}$, for some $k_{\varepsilon} \in \mathbb{N}^{*}$, we deduce that

$$
\int_{I_{1}^{(j)}} \varphi_{k}(x)^{2} \mathrm{~d} x \geq \frac{\varepsilon}{4}\left(c_{k, j}^{2}+d_{k, j}^{2}\right) .
$$

By summing this estimate on $j$ and using the normalization estimate (7.29), we obtain (7.27) for $k \geq k_{\varepsilon}$. The proof is complete since for $k \leq k_{\varepsilon}$,

$$
\sum_{j=1}^{N} \alpha_{1}^{(j)} \int_{I_{1}^{(j)}} \varphi_{k}(x)^{2} \mathrm{~d} x>0 .
$$

The analysis of the condition (7.27) in some particular cases reveals that the condition of the above proposition is far from being optimal but in its full generality we cannot easily obtain a weaker condition.

As in the previous subsection one easily shows (see the proof of Lem. 7.11) that the operators $A$ and $B=$ $\left(B_{1} B_{2}\right) \in \mathcal{L}\left(U, V^{\prime}\right)$ where $U=U_{1} \times U_{2}$ satisfy the assumption (4.1). 
In conclusion applying either Theorem 4.4 or Theorem 5.3, we obtain:

Proposition 7.20. Assume that $\alpha_{2}^{(j)}<\alpha_{1}^{(j)}$ for all $j=1, \ldots, N$ and that the standard gap condition (6.4) holds and that all eigenvalues are simple (i.e., $l_{k}=1$ ). Then

(i) The energy of system (7.25) decays exponentially if (7.27) holds.

(ii) The energy of system (7.25) decays like $t^{-\frac{1}{m}}$ if (7.28) holds.

\section{REFERENCES}

[1] C. Abdallah, P. Dorato, J. Benitez-Read and R. Byrne, Delayed positive feedback can stabilize oscillatory systems, in $A C C^{\prime}$ 93 (American Control Conference), San Francisco (1993) 3106-3107.

[2] K. Ammari and M. Tucsnak, Stabilization of Bernoulli-Euler beams by means of a pointwise feedback force. SIAM J. Control Optim. 39 (2000) 1160-1181 (electronic).

[3] K. Ammari and M. Tucsnak, Stabilization of second order evolution equations by a class of unbounded feedbacks. ESAIM: COCV 6 (2001) 361-386 (electronic).

[4] K. Ammari, E.M. Ait Ben Hassi, S. Boulite and L. Maniar, Feedback stabilization of a class of evolution equations with delay. J. Evol. Eq. (Submitted).

[5] W. Arendt and C.J.K. Batty, Tauberian theorems and stability of one-parameter semigroups. Trans. Amer. Math. Soc. 305 (1988) 837-852.

[6] C. Baiocchi, V. Komornik and P. Loreti, Ingham-Beurling type theorems with weakened gap conditions. Acta Math. Hungar. 97 (2002) 55-95.

[7] R. Dáger and E. Zuazua, Wave propagation, observation and control in 1-d flexible multi-structures, Mathématiques 83 Applications 50. Springer-Verlag, Berlin (2006).

[8] R. Datko, Not all feedback stabilized hyperbolic systems are robust with respect to small time delays in their feedbacks. SIAM J. Control Optim. 26 (1988) 697-713.

[9] R. Datko, Two examples of ill-posedness with respect to time delays revisited. IEEE Trans. Automat. Contr. 42 (1997) 511-515.

[10] R. Datko, J. Lagnese and M.P. Polis, An example on the effect of time delays in boundary feedback stabilization of wave equations. SIAM J. Control Optim. 24 (1986) 152-156.

[11] K.P. Hadeler, Delay equations in biology, in Functional differential equations and approximation of fixed points, Lect. Notes Math. 730, Springer, Berlin (1979) 136-156.

[12] J. Hale and S. Verduyn Lunel, Introduction to functional differential equations, Applied Mathematical Sciences 99. Springer (1993).

[13] A.E. Ingham, Some trigonometrical inequalities with applications to the theory of series. Math. Z. 41 (1936) 367-379.

[14] I. Lasiecka, R. Triggiani and P.-F. Yao. Inverse/observability estimates for second-order hyperbolic equations with variable coefficients. J. Math. Anal. Appl. 235 (1999) 13-57.

[15] H. Logemann, R. Rebarber and G. Weiss, Conditions for robustness and nonrobustness of the stability of feedback systems with respect to small delays in the feedback loop. SIAM J. Control Optim. 34 (1996) 572-600.

[16] S. Nicaise and C. Pignotti, Stability and instability results of the wave equation with a delay term in the boundary or internal feedbacks. SIAM J. Control Optim. 45 (2006) 1561-1585 (electronic).

[17] S. Nicaise and J. Valein, Stabilization of the wave equation on 1-D networks with a delay term in the nodal feedbacks. Netw. Heterog. Media 2 (2007) 425-479 (electronic).

[18] A. Pazy, Semigroups of linear operators and applications to partial differential equations. Appl. Math. Sci. 44 (1983).

[19] R. Rebarber, Exponential stability of coupled beams with dissipative joints: a frequency domain approach. SIAM J. Control Optim. 33 (1995) 1-28.

[20] R. Rebarber and S. Townley, Robustness with respect to delays for exponential stability of distributed parameter systems. SIAM J. Control Optim. 37 (1999) 230-244.

[21] I.H. Suh and Z. Bien, Use of time delay action in the controller design. IEEE Trans. Automat. Contr. 25 (1980) 600-603.

[22] M. Tucsnak and G. Weiss, How to get a conservative well-posed linear system out of thin air. II. Controllability and stability. SIAM J. Control Optim. 42 (2003) 907-935.

[23] G.Q. Xu, S.P. Yung and L.K. Li, Stabilization of wave systems with input delay in the boundary control. ESAIM: COCV 12 (2006) 770-785 (electronic). 